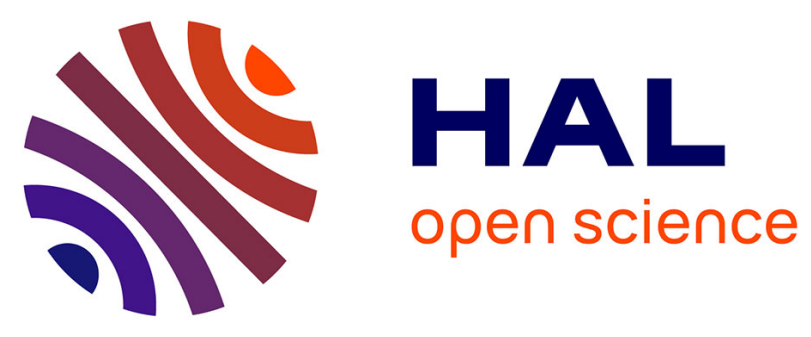

\title{
State and output feedback fuzzy variable structure controllers for multivariable nonlinear systems subject to input nonlinearities
}

\author{
Abdesselem Boulkroune, Mohammed M'Saad, Mondher Farza
}

\section{- To cite this version:}

Abdesselem Boulkroune, Mohammed M'Saad, Mondher Farza. State and output feedback fuzzy variable structure controllers for multivariable nonlinear systems subject to input nonlinearities. International Journal of Advanced Manufacturing Technology, 2014, Vol. 71, pp.539-556. 10.1007/s00170013-5453-4 . hal-01063919

\section{HAL Id: hal-01063919 \\ https://hal.science/hal-01063919}

Submitted on 15 Sep 2014

HAL is a multi-disciplinary open access archive for the deposit and dissemination of scientific research documents, whether they are published or not. The documents may come from teaching and research institutions in France or abroad, or from public or private research centers.
L'archive ouverte pluridisciplinaire HAL, est destinée au dépôt et à la diffusion de documents scientifiques de niveau recherche, publiés ou non, émanant des établissements d'enseignement et de recherche français ou étrangers, des laboratoires publics ou privés. 


\title{
State and output feedback fuzzy variable structure controllers for multivariable nonlinear systems subject to input nonlinearities
}

\author{
Abdesselem Boulkroune • Mohammed Msaad • \\ Mondher Farza
}

\begin{abstract}
This paper presents three fuzzy adaptive controllers for a class of uncertain multivariable nonlinear systems with both sector nonlinearities and dead zones: two first controllers are state feedbacks and the last controller is an output feedback. The design of the first controller concerns systems with symmetric and positive definite control-gain matrix, while the second control design is extended to the case of nonsymmetric control-gain matrix thanks to an appropriate decomposition, namely the product of a symmetric positive definite matrix, a diagonal matrix with diagonal entries +1 or -1 , and a unity upper triangular matrix. The third controller is an output feedback extension of the second controller. In this controller, a high-gain observer is incorporated to estimate the unmeasurable states. An appropriate adaptive fuzzy logic system is used to reasonably approximate the uncertain functions. A Lyapunov approach is adopted to derive the parameter adaptation laws and prove the stability of those control systems as well as the exponential convergence of their underlying tracking errors within an adjustable region. The effectiveness of the proposed fuzzy adaptive controllers is illustrated through simulation results.
\end{abstract}

\footnotetext{
A. Boulkroune ( $\square$ )

Department of Automatic, Faculty of Engineering Sciences, Jijel University, BP. 98, Ouled-Aissa 18000, Jijel, Algeria

e-mail: boulkroune2002@yahoo.fr

M. Msaad • M. Farza

GREYC, UMR 6072 CNRS, Université de Caen, ENSICAEN, 6 Bd Maréchal Juin, 14050 Caen Cedex, France

M. Msaad

e-mail:msaad@greyc.ensicaen.fr

M. Farza

e-mail:mfarza@greyc.ensicaen.fr
}

Keywords Adaptive fuzzy control · Variable structure control · High-gain observer · Multivariable nonlinear systems $\cdot$ Sector nonlinearity $\cdot$ Dead zone

\section{Introduction}

The design of robust adaptive controllers for multivariable unknown nonlinear systems remains one of the most challenging tasks in the area of control systems. Based on the universal approximation theorem [1], some adaptive fuzzy control systems [2-11] have been developed for a class of multivariable nonlinear uncertain systems. The stability of the underlying control systems has been investigated using a Lyapunov approach. The robustness issues with respect to the approximation error and external disturbances have been enhanced by appropriately modifying the available adaptive fuzzy controllers. The cornerstone of such a modification consists in a robust compensator which is conceived using a sliding mode control design $[3,5,6,8,10,11]$ or an $H^{\infty}$-based robust control design $[2,4,7,9]$. A key assumption in the available fuzzy adaptive control systems [2-11] is that the actuator dynamics may be reasonably approximated by a linear model. This is more an exception than a rule in the engineering practice.

The control problem of uncertain multivariable systems with nonlinear input channels has received a remarkable attention because of those ubiquitous actuator nonlinearities, namely saturation, quantization, backlash, dead zone, and so on $[12,13]$. It is well known that the existence of input nonlinearities may lead to poor performance or even instability of the control system. It is thereby more advisable to take into account the actuator nonlinearities in the control design as well as the stability analysis. Decentralized variable structure controllers have been proposed in [14-16] for a class of systems with input sector nonlinearities and/or dead zones. 
In $[13,17]$, the authors designed sliding-mode control systems for nonlinear multivariable systems subject to both sector nonlinearities and dead zones. The underlying results suffer from two fundamental limitations. Firstly, the considered class of systems is relatively reduced. Secondly, the gain reduction tolerances of the nonlinear dead zones and upper bounds of uncertain nonlinear functions are required to be known.

More recently, adaptive neural or fuzzy control systems have been respectively proposed for a particular class of multivariable nonlinear systems with unknown dead zones and gain signs in [18] and [19]. These contributions suffer from two restrictive modelling assumptions motivated by technical purposes regarding the stability analysis and control design. The first one consists in assuming a lower triangular structure for the system under control while the second one concerns the boundedness of the high-frequency control gains. Moreover, an adaptive fuzzy control for a class of multivariable nonlinear systems with unknown dead zones has been designed in [20]. Note that, in [18-20], simple dead zones having linear and nonlinear functions outside the dead band have been considered.

In this paper, one aims at designing three fuzzy adaptive controllers for three different classes of uncertain nonlinear multivariable systems containing both sector nonlinearities and dead zones, namely:

- Multiple input, multiple output (MIMO) systems with a symmetric positive definite control-gain matrix and measurable states

- MIMO systems with a nonsymmetric control-gain matrix having non-zero-leading principal minors and measurable states, and

- MIMO systems with a nonsymmetric control-gain matrix having non-zero-leading principal minors but with states not being available for measurement.

Though this work borrows from the available results, it presents a fundamental contribution to the fuzzy adaptive control of uncertain multivariable nonlinear systems from an applicability point of view. The main contributions of this paper are emphasized below:

(a) Unlike in contributions [13, 17], the class of the considered systems is relatively larger and the gain reduction tolerances of the nonlinear dead zones and the upper bounds on uncertain nonlinear functions are not assumed to be known. These bounds are indeed estimated using adaptive fuzzy systems.

(b) And compared with contributions [18-20], there are three features that are of practical interest. Firstly, the considered class of systems is larger as the modelling assumptions made in $[18,19]$ are relatively restrictive, namely lower triangular control structure with bounded high-frequency control gains. Such modelling requirements are mainly motivated by stability analysis and control design purposes. Secondly, the considered model of the input nonlinearity includes sector nonlinearities and dead zones and is hence relatively larger than the one considered in [18-20]. Thirdly, the nonsymmetric control-gain matrix is appropriately decomposed into a product of a symmetric definite positive matrix, a diagonal matrix with +1 or -1 on the diagonal and a unity upper triangular matrix. It is worth noticing that the diagonal matrix elements are nothing than the ratios of the signs of the leading principal minors of the control-gain matrix.

(c) Moreover, unlike in the references [13, 17-20], in our third controller, the system states are assumed to be unknown. In fact, a high-gain observer is designed to estimate those missing states.

\section{Notation and problem statement}

Consider the following class of nonlinear multivariable systems described by:

$$
\begin{gathered}
y_{1}^{\left(r_{1}\right)}=f_{1}(x)+\sum_{j=1}^{p} g_{1 j}(x) \Phi_{j}\left(u_{j}\right), \\
\vdots \\
y_{p}^{\left(r_{p}\right)}=f_{p}(x)+\sum_{j=1}^{p} g_{p j}(x) \Phi_{j}\left(u_{j}\right),
\end{gathered}
$$

where $x_{i}=\left[x_{i 1}, x_{i 2}, \ldots, x_{i r_{i}}\right]^{T}=\left[y_{i}, \dot{y}_{i}, \ldots, y_{i}^{\left(r_{i}-1\right)}\right]^{T}$, for $i=1$, $\ldots, p$, is the state vector of the subsystem $i, x=\left[x_{1}^{T}, x_{2}^{T}\right.$, $\left.\ldots, x_{p}^{T}\right]^{T} \in R^{r}$ is the overall state vector with $r=r_{1}+\ldots+r_{p}$, $u=\left[u_{1}, \ldots, u_{p}\right]^{T} \in R^{p}$ is the control input vector, $y=\left[y_{1}\right.$, $\left.\ldots, y_{p}\right]^{T} \in R^{p}$ is the output vector, $f_{i}(x)$ and $g_{i j}(x), i, j=1, \ldots$, $p$ are unknown continuous nonlinear functions, and $\Phi(u)=\left[\Phi_{1}\left(u_{1}\right), \Phi_{2}\left(u_{2}\right), \ldots, \Phi_{p}\left(u_{p}\right)\right]^{T}$ is a nonlinear input function vector satisfying some properties which will be given in Section 2.2.

Let us denote

$$
\begin{gathered}
y^{(r)}=\left[y_{1}^{\left(r_{1}\right)} \ldots y_{p}^{\left(r_{p}\right)}\right]^{T}, \quad F(x)=\left[f_{1}(x) \ldots f_{p}(x)\right]^{T}, \\
G(x)=\left[\begin{array}{ccc}
g_{11}(x) & \ldots & g_{1 p}(x) \\
\vdots & \ddots & \vdots \\
g_{p 1}(x) & \ldots & g_{p p}(x)
\end{array}\right]
\end{gathered}
$$

Then, the system (1) can be rewritten in the following compact form:

$y^{(r)}=F(x)+G(x) \Phi(u)$

where $F(.) \in R^{p}$ and $G(.) \in R^{p \times p}$. 
Remark 1 The system (1) represents a class of MIMO nonlinear systems with input nonlinearities $\Phi(u)$ (i.e., with dead zone and sector nonlinearities). It is worth noticing that the input nonlinearities exist widely in the practical control systems, such as mechanical connections, hydraulic servo-valves, piezo-positioners, and biomedical systems, but they are generally neglected in the control design for simplicity purposes. Also, the MIMO system (1) without input nonlinearities has been considered by many literatures [2-11] and is of higher practical significance. Note that many practical systems can be represented in the form of the MIMO nonlinear systems (1) such as robotic systems, electrical machines, mechanical systems, and chaotic systems.

The objective of this paper is to design a stable adaptive control system allowing the system output vector $y$ to follows a specified desired trajectory $y_{d}=\left[y_{d 1}, \ldots, y_{d p}\right]^{T} \in R^{p}$. We assume that the vector $x_{d}=\left[y_{d 1}, \dot{y}_{d 1}, \ldots, y_{d 1}^{\left(r_{1}-1\right)}, y_{d 1}^{\left(r_{1}\right)}, \ldots, y_{d p}\right.$, $\left.\dot{y}_{d p}, \ldots, y_{d p}^{\left(r_{p}-1\right)}, y_{d p}^{\left(r_{p}\right)}\right]^{T}$ is continuous, bounded, and available for measurement. Then, $x_{d} \in \Omega_{x_{d}} \subset R^{r+p}$ with $\Omega_{x_{d}}$ is a known compact set.

Let us define the tracking error as

$$
\begin{gathered}
e_{1}=y_{1}-y_{d 1} \\
\vdots \\
e_{p}=y_{p}-y_{d p}
\end{gathered}
$$

and the filtered tracking error as

$S=\left[S_{1}, \ldots, S_{p}\right]^{T}$

with

$S_{i}=\left[\frac{d}{d t}+\lambda_{i}\right]^{r_{i}-1} e_{i}$ for $\lambda_{i}>0, \quad \forall i=1, \ldots, p$

Then, one can rewrite Eq. (5) as follows

$S_{i}=\lambda_{i}^{r_{i}-1} e_{\mathrm{i}}+\left(r_{i}-1\right) \lambda_{i}^{r_{i}-2} \dot{e}_{\mathrm{i}}+\cdots+\left(r_{i}-1\right) \lambda_{i} e_{i}^{\left(r_{i}-2\right)}+e_{i}^{\left(r_{i}-1\right)}$

with $i=1, \ldots, p$

Notice that if one chooses $\lambda_{i}>0$, with $i=1, \ldots, p$, then the roots of the polynomial $H_{i}(s)=\lambda_{i}^{r_{i}-1}+\left(r_{i}-1\right) \lambda_{i}^{r_{i}-2} s+\cdots+$ $\left(r_{i}-1\right) \lambda_{i} s^{r_{i}-2}+s^{r_{i}-1}$ related to the characteristic equation of $S_{i}=0$ are all in the open left-half plane [21].

The relation (6) can be rewritten in the following compact form

$S_{i}=C_{i}^{T} E_{i}$ with

$E_{i}=\left[e_{i} \dot{e}_{i} \ldots e_{i}^{\left(r_{i}-2\right)} e_{i}^{\left(r_{i}-1\right)}\right]^{T}$

$C_{i}^{T}=\left[\begin{array}{llll}\lambda_{i}^{r_{i}-1}\left(r_{i}-1\right) \lambda_{i}^{r_{i}-2} & \ldots & \left(r_{i}-1\right) \lambda_{i} & 1\end{array}\right]$

Consequently, the vector $S$ takes the form:

$S=C^{T} E$

where

$C^{T}=\operatorname{diag}\left[\begin{array}{lll}C_{1}^{T} C_{2}^{T} & \ldots & C_{p}^{T}\end{array}\right]_{(p \times r)}$

$E=\left[\begin{array}{lll}E_{1}^{T} E_{2}^{T} & \ldots & E_{p}^{T}\end{array}\right]_{(r \times 1)}^{T}$

And the dynamic of $S_{i}$ is described by:

$\dot{S}_{i}=C_{r i}^{T} E_{i}+e_{i}^{\left(r_{i}\right)}$, and $i=1, \ldots, p$

with

$C_{r i}^{T}=\left[\begin{array}{lllll}0 & \lambda_{i}^{r_{i}-1} & \left(r_{i}-1\right) \lambda_{i}^{r_{i}-2} \ldots & 0.5\left(r_{i}-1\right)\left(r_{i}-2\right) \lambda_{i}^{2} & \left(r_{i}-1\right) \lambda_{i}\end{array}\right]$

The dynamic of $S$ can be written into the following compact form

$\dot{S}=C_{r}^{T} E+e^{(r)}$

where

$C_{r}^{T}=\operatorname{diag}\left[C_{r 1}^{T} C_{r 2}^{T} \quad \ldots C_{r p}^{T}\right]_{(p \times r)}$

$e^{(r)}=\left[\begin{array}{llll}e_{1}^{\left(r_{1}\right)} & e_{2}^{\left(r_{2}\right)} & \ldots & e_{p}^{\left(r_{p}\right)}\end{array}\right]^{T}$

with

$e^{(r)}=y^{(r)}-y_{d}^{(r)}$

where $y^{(r)}=\left[\begin{array}{llll}y_{1}^{\left(r_{1}\right)} & y_{2}^{\left(r_{2}\right)} & \ldots & y_{p}^{\left(r_{p}\right)}\end{array}\right]^{T}$ is previously defined and

$y_{d}^{(r)}=\left[\begin{array}{llll}y_{d 1}^{\left(r_{1}\right)} & y_{d 2}^{\left(r_{2}\right)} & \ldots & y_{d p}^{\left(r_{p}\right)}\end{array}\right]^{T}$,

From (18), one can write (15) as follows

$\dot{S}=C_{r}^{T} E+y^{(r)}-y_{d}^{(r)}$ 
Thereafter, (20) will be used in the development of the proposed controllers and the stability analysis.

\subsection{Description of the fuzzy logic system}

In this paper, a zero-order Takagi-Sugeno fuzzy system will be used to approximate unknown nonlinear functions. The basic configuration of a fuzzy logic system consists of a fuzzifier, a fuzzy inference engine, and a defuzzifier, as shown in Fig. 1. The fuzzy inference engine uses the fuzzy IF-THEN rules to perform a mapping from an input vector $\underline{x}^{T}=\left[x_{1}, x_{2}, \ldots, x_{n}\right]$ $\in R^{n}$ to an output $\widehat{f} \in R$. The $i$ th fuzzy rule is written as

$R^{(i)}:$ if $x_{1}$ is $A_{1}^{i}$ and... and $x_{n}$ is $A_{n}^{i}$ then $\widehat{f}$ is $f^{i}$

where $A_{1}^{i}, A_{2}^{i}, \ldots$, and $A_{n}^{i}$ are fuzzy sets and $f^{i}$ is the fuzzy singleton for the output in the $i$ th rule. By using the singleton fuzzifier, the product inference and the center-average defuzzifier, the output of the fuzzy system can be expressed as follows:

$$
\begin{aligned}
\widehat{f}(\underline{x}) & =\frac{\sum_{i=1}^{m} f^{i}\left(\prod_{j=1}^{n} \mu_{A_{j}^{i}}\left(x_{j}\right)\right)}{\sum_{i=1}^{m}\left(\prod_{j=1}^{n} \mu_{A_{j}^{i}}\left(x_{j}\right)\right)} \\
& =\theta^{T} \psi(\underline{x})
\end{aligned}
$$

where $\mu_{A_{j}^{i}}\left(x_{j}\right)$ is the degree of membership of $x_{j}$ to $A_{j}^{i}, m$ is the number of fuzzy rules, $\theta^{T}=\left[f^{1}, f^{2}, \ldots, f^{m}\right]$ is the adjustable parameter vector (composed of consequent parameters) and $\psi^{T}=\left[\psi^{1} \psi^{2} \ldots \psi^{m}\right]$ with

$\psi^{i}(\underline{x})=\frac{\left(\prod_{j=1}^{n} \mu_{A_{j}^{i}}\left(x_{j}\right)\right)}{\sum_{i=1}^{m}\left(\prod_{j=1}^{n} \mu_{A_{j}^{i}}\left(x_{j}\right)\right)}$

being the fuzzy basis function $(F B F)$. Throughout the paper, it is assumed that the FBFs are selected so that there is always at least one active rule [1, 22-24], i.e., $\sum_{i=1}^{m}\left(\prod_{j=1}^{n} \mu_{A_{j}^{i}}\left(x_{j}\right)\right)>0$.

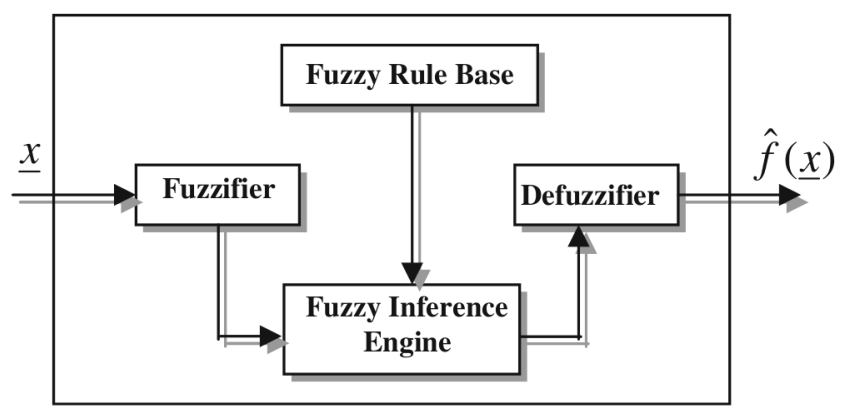

Fig. 1 The basic configuration of a fuzzy logic system
It is worth noting that the fuzzy system (22) is commonly used in control applications. Following the universal approximation results, the fuzzy system (22) is able to approximate any nonlinear smooth function on a compact operating space to an arbitrary degree of accuracy [1]. Of particular importance, it is assumed that the structure of the fuzzy system, namely the pertinent inputs, the number of membership functions for each input and the number of rules, and the membership function parameters are properly specified beforehand. The consequent parameters $\theta$ are then determined by appropriate parameter adaptation algorithms.

\subsection{Input nonlinearity}

The mathematical model of the input nonlinearity (i.e., the sector nonlinearity and dead zone) under consideration is described by

$\Phi_{i}\left(u_{i}\right)= \begin{cases}\phi_{i+}\left(u_{i}\right)\left(u_{i}-u_{i+}\right), & u_{i}>u_{i+}, \\ 0, & -u_{i-} \leq u_{i} \leq u_{i+}, \\ \phi_{i-}\left(u_{i}\right)\left(u_{i}+u_{i-}\right), & u_{i}<-u_{i-},\end{cases}$

where $\phi_{i+}\left(u_{i}\right)>0$ and $\phi_{i-}\left(u_{i}\right)>0$ are nonlinear functions of $u_{i}$, and $u_{i+}>0$ and $u_{i-}>0$ are known constants. The involved modelling assumption is

Assumption 1 The input nonlinearity $\Phi_{i}\left(u_{i}\right)$ satisfies the following important properties:

$$
\begin{aligned}
& \left(u_{i}-u_{i+}\right) \Phi_{i}\left(u_{i}\right) \geq m_{i+}^{*}\left(u_{i}-u_{i+}\right)^{2}, \text { for } u_{i}>u_{i+}, \\
& \left(u_{i}+u_{i-}\right) \Phi_{i}\left(u_{i}\right) \geq m_{i-}^{*}\left(u_{i}+u_{i-}\right)^{2}, \text { for } u_{i}<-u_{i-},
\end{aligned}
$$

where $m_{i+}^{*}$ and $m_{i-}^{*}$ are unknown constants which are called "gain reduction tolerances".

It is worth mentioning that the models (24)-(25) allows to consider both dead zones and sector nonlinearities with reduced prior knowledge. Indeed, the gain reduction tolerances $m_{+}^{*}$ and $m_{-}^{*}$ are unknown, unlike in [13] to [17], and the input nonlinearity is only characterized throughout the property (25) together with the knowledge of the constants $u_{+}$and $u_{-}$. By the way, notice that the dead zone considered in the contribution $[19,20]$ is a particular case of the above general form.

\section{Design of state feedback fuzzy adaptive controllers}

In this section, two state feedback fuzzy adaptive variable structure control designs are proposed up to an assumption on the structure of the control-gain matrix. In the first one, the control-gain matrix is assumed to be symmetric and positive definite. Such an assumption is relaxed in the second design where the control-gain matrix may not be symmetric and 
positive definite provided that the signs of its leading principal minors are known.

Here, for both controllers, we assume that the system outputs $y_{i}, i=1, \ldots, p$, and its $j$ th derivatives (for $j=1, \ldots, r_{i}-1$ ) are available for measurements, i.e., all state information are available.

\subsection{The first state feedback fuzzy adaptive controller}

In the following, we present a fuzzy adaptive variable-structure controller for the class of system (2) under the following assumption on the control-gain matrix.

Assumption 2 The control-gain matrix is symmetric, positive definite, and of class $C^{l}$ with the following property

$\partial g_{i j}(x) / \partial y_{i}^{\left(r_{i}-1\right)}=0 \quad \forall i=1,2, \ldots, p$ and $j=1,2, \ldots, p$.

Such an assumption is not too restrictive in nature as there are many physical mechanical and electrical systems whose dynamics can be described by Eq. (2) with symmetric, positive definite, and class $C^{1}$ control-gain matrix [25]. The positive definite property of the control-gain matrix, which is closely related to the system controllability, has been already required in recent fundamental contributions on adaptive (fuzzy or neural) control of multivariable systems. Moreover, the required property on the partial derivatives of the control-gain matrix ensures that the time derivative of its inverse, i.e., $d G^{-1}(x) / d t$, depends only on the state vector $x$ (i.e., it ensures that $d G^{-1}(x) /$ $d t$ does not depend on the system input $u$ ). Notice that the following fundamental results could be adapted, up to some appropriate modifications, to the case where the control-gain matrix is symmetric and negative definite.

By substituting (2) into (20), the dynamics of $S$ become

$\dot{S}=C_{r}^{T} E+F(x)+G(x) \Phi(u)-y_{d}^{(r)}$

Letting $G_{1}(x)=G^{-1}(x)$, one has

$G_{1}(x) \dot{S}=G_{1}(x)\left[C_{r}^{T} E-y_{d}^{(r)}+F(x)\right]+\Phi(u)$

For stability analysis and control design simplicity purposes, it is more advisable to rewrite the dynamics of $S$ as follows

$$
\begin{aligned}
\frac{1}{2} \dot{G}_{1}(x) S+G_{1}(x) \dot{S} & =\frac{1}{2} \dot{G}_{1}(x) S+G_{1}(x)\left[C_{r}^{T} E-y_{d}^{(r)}+F(x)\right]+\Phi(u) \\
& =\alpha(x, v, S)+\Phi(u)
\end{aligned}
$$

with

$$
\begin{aligned}
\alpha(x, v, S) & =\left[\alpha_{1}(x, v, S), \alpha_{2}(x, v, S), \ldots, \alpha_{p}(x, v, S)\right]^{T} \\
& =(1 / 2) \dot{G}_{1}(x) S+G_{1}(x)[v+F(x)]
\end{aligned}
$$

and

$v=C_{r}^{T} E-y_{d}^{(r)}$

This form makes it possible to introduce a further assumption that has to be made to get the fundamental result we are concerned by, namely

Assumption 3 There exists an unknown continuous positive function $\bar{\alpha}_{i}(x)$ such that $\left|\alpha_{i}(x, v, S)\right| \leq \eta \bar{\alpha}_{i}(x) \quad \forall x \in \Omega_{x} \subset R^{r}$ with $\eta=\min _{i}\left\{\eta_{i}\right\}$ where $\eta_{i}=\min \left\{m_{i+}^{*}, m_{i-}^{*}\right\}$

Notice that such an assumption is not restrictive since $\bar{\alpha}_{i}(x)$ is assumed to be unknown. Moreover, since $v$ and $S$ are functions of $\left(x, x_{d}\right), x_{d} \in L_{\infty}$ and $\alpha_{i}(x, v, S)$ is a continuous function, such a function always exists. The unknown continuous nonlinear function $\bar{\alpha}_{i}(x)$ can be hence approximated, on a compact set $\Omega_{x}$, by the fuzzy systems (22) as follows:

$\widehat{\bar{\alpha}}_{i}(x, \theta)=\theta_{i}^{T} \psi_{i}(x)$

where $\psi_{i}(x)$ is the FBF vector, which is fixed a priori by the designer, and $\theta_{i}$ is the adjustable parameter vector of the fuzzy system (composed of consequent parameters). Its adaptive law will be designed later.

According to $[1,26,27]$, the unknown continuous function $\bar{\alpha}_{i}(x)$ can be optimally approximated as

$$
\begin{aligned}
\bar{\alpha}_{i}(x) & =\widehat{\bar{\alpha}}_{i}\left(x, \theta_{i}^{*}\right)+\delta_{i}(x) \\
& =\theta_{i}^{* T} \psi_{i}(x)+\delta_{i}(x)
\end{aligned}
$$

where $\delta_{i}(x)$ is the fuzzy approximation error and $\theta_{i}^{*}$ is the optimal parameter vector defined by

$\theta_{i}^{*}=\underset{\theta_{i}}{\arg \min }\left[\sup _{x \in \Omega_{x}}\left|\bar{\alpha}_{i}(x)-\widehat{\bar{\alpha}}_{i}\left(x, \theta_{i}\right)\right|\right]$

Note that this vector is mainly introduced for analysis purposes as its value is not needed when implementing the controller [28-31]. As in the open literature [1, 25-27], one assumes that the used fuzzy systems do not violate the universal approximator property on the compact set $\Omega_{x}$, which is assumed large enough so that the input vector of the fuzzy system remains in $\Omega_{x}$ for the control system. It is hence reasonable to assume that the fuzzy approximation error is bounded for all $x \in \Omega_{x}$, i.e.,

$\left|\delta_{i}(x)\right| \leq \bar{\delta}_{i}, \quad \forall x \in \Omega_{x}$, 
where $\bar{\delta}_{i}$ is an unknown constant. From the above analysis, one has

$$
\begin{aligned}
\widehat{\bar{\alpha}}_{i}\left(x, \theta_{i}\right)-\bar{\alpha}_{i}(x) & =\widehat{\bar{\alpha}}_{i}\left(x, \theta_{i}\right)-\widehat{\bar{\alpha}}_{i}\left(x, \theta_{i}^{*}\right)+\widehat{\bar{\alpha}}_{i}\left(x, \theta_{i}^{*}\right)-\bar{\alpha}_{i}(x), \\
& =\widehat{\bar{\alpha}}_{i}\left(x, \theta_{i}\right)-\widehat{\bar{\alpha}}_{i}\left(x, \theta_{i}^{*}\right)-\delta_{i}(x), \\
& ={\widetilde{\theta_{i}}}^{T} \psi_{i}(x)-\delta_{i}(x)
\end{aligned}
$$

where $\widetilde{\theta}_{i}=\theta_{i}-\theta_{i}^{*}$ is the parameter estimation error.

The following fuzzy adaptive variable structure controller is proposed to achieve the control objective.

$u_{i}= \begin{cases}-\rho_{i}(t) \operatorname{sign}\left(S_{i}\right)-u_{i^{-}}, & S_{i}>0, \\ 0, & S_{i}=0, \\ -\rho_{i}(t) \operatorname{sign}\left(S_{i}\right)+u_{i+}, & S_{i}<0,\end{cases}$

with

$$
\begin{gathered}
\rho_{i}(t)=k_{0 i}+k_{1 i}\left|S_{i}\right|+\theta_{i}^{T} \psi_{i}(x), \quad \forall i=1, \ldots, p \\
\dot{k}_{0 i}=-\gamma_{0 i} \sigma_{0 i} k_{0 i}+\gamma_{0 i}\left|S_{i}\right| \text { for } k_{0 i}(0)>0
\end{gathered}
$$

$\dot{\theta}_{i}=-\gamma_{1 i} \sigma_{1 i} \theta_{i}+\gamma_{1 i}\left|S_{i}\right| \psi_{i}(x)$ with $\theta_{i j}(0)>0$

where $\gamma_{0 i}, \gamma_{1 i}, \sigma_{0 i}, \sigma_{1 i}, k_{1 i}>0$ are design constants, and $k_{0 i}$ and $\theta_{i}$ are the online estimates of the uncertain terms $k_{0 i}^{*}=\bar{\delta}_{i}$ and $\theta_{i}^{*}$, respectively. It is important to remark that if $k_{0 i}(0)>0$ and $\theta_{i j}(0)>0$ for $i=1, \ldots, p$ and $j=1, \ldots, m$, it follows from the adaptive laws (34-35) that their respective solutions satisfy $k_{0 i}(t)>0$ and $\theta_{i j}(t)>0$, for $t>0$.

Multiplying (28) by $S^{T}$ and using Assumption 3, one has

$$
\begin{aligned}
\frac{1}{2 \eta} S^{T} \dot{G}_{1}(x) S+\frac{1}{\eta} S^{T} G_{1}(x) \dot{S} & =\frac{1}{\eta} S^{T} \alpha(x, v, S)+\frac{1}{\eta} S^{T} \Phi(u) \\
& \leq \sum_{i=1}^{p}\left|S_{i}\right| \bar{\alpha}_{i}(x)+\frac{1}{\eta} S^{T} \Phi(u)
\end{aligned}
$$

And using (32) and (36) yields

$$
\begin{aligned}
\frac{1}{2 \eta} S^{T} \dot{G}_{1}(x) S & +\frac{1}{\eta} S^{T} G_{1}(x) \dot{S} \leq \sum_{i=1}^{p}\left|S_{i}\right| \bar{\alpha}_{i}(x)+\frac{1}{\eta} S^{T} \Phi(u) \\
& \leq-\sum_{i=1}^{p}\left|S_{i}\right| \widetilde{k}_{0 i}-\sum_{i=1}^{p}\left|S_{i}\right| \widetilde{\theta}_{i}^{T} \psi_{i}(x)+\sum_{i=1}^{p}\left|S_{i}\right| k_{0 i} \\
& +\sum_{i=1}^{p}\left|S_{i}\right| \theta_{i}^{T} \psi_{i}(x)+\frac{1}{\eta} S^{T} \Phi(u)
\end{aligned}
$$

where $\widetilde{\theta}_{i}=\theta_{i}-\theta_{i}^{*}$ and $\widetilde{k}_{0 i}=k_{0 i}-k_{0 i}^{*}=k_{0 i}-\bar{\delta}_{i}$.

This allows to state the fundamental result concerning the first fuzzy adaptive control system.
Theorem 1 Consider the system (2) subject to Assumptions 1-3. Then, the control law defined by Eqs. (33) to (35) guarantees the following properties:

- All the variables involved in the closed-loop system are uniformly ultimately bounded (UUB).

- The filtered tracking errors $S_{i}$ of the control system exponentially converge to an adjustable domain defined by:

$$
\Omega_{S_{i}}=\left\{S_{i}|| S_{i} \mid \leq\left(\frac{2 \eta \pi_{1}}{\sigma_{g 1} \mu_{1}}\right)^{1 / 2}\right\}
$$

where $\pi_{1}, \mu_{1}$ and $\sigma_{g 1}$ will be defined later.

Proof Let us consider the following Lyapunov function candidate:

$$
V=\frac{1}{2 \eta} S^{T} G_{1}(x) S+\frac{1}{2} \sum_{i=1}^{p} \frac{1}{\gamma_{0 i}} \widetilde{k}_{0 i}^{2}+\frac{1}{2} \sum_{i=1}^{p} \frac{1}{\gamma_{1 i}} \widetilde{\theta}_{i}^{T} \widetilde{\theta}_{i} .
$$

Its time derivative is given by

$$
\dot{V}=\frac{1}{\eta} S^{T} G_{1}(x) \dot{S}+\frac{1}{2 \eta} S^{T} \dot{G}_{1}(x) S+\sum_{i=1}^{p} \frac{1}{\gamma_{0 i}} \widetilde{k}_{0 i} \dot{k}_{0 i}+\sum_{i=1}^{p} \frac{1}{\gamma_{1 i}} \widetilde{\theta}_{i}^{T} \dot{\theta}_{i}
$$

One can easily show from (25) and (33) that

$$
\begin{array}{r}
u_{i}<-u_{i-} \text { for } S_{i}>0 \Rightarrow\left(u_{i}+u_{i^{-}}\right) \Phi_{i}\left(u_{\mathrm{i}}\right) \\
=m_{i^{-}}^{*}\left(u_{i}+u_{i^{-}}\right)^{2} \geq \eta\left(u_{i}+u_{i^{-}}\right)^{2}
\end{array}
$$

and

$$
\begin{array}{r}
u_{i}>u_{i+} \text { for } S_{i}<0 \Rightarrow\left(u_{i}-u_{i+}\right) \Phi_{i}\left(u_{i}\right) \\
=m_{i+}^{*}\left(u_{i}-u_{i+}\right)^{2} \geq \eta\left(u_{i}-u_{i+}\right)^{2}
\end{array}
$$

From this analysis and (33), one can also conclude that for $S_{i}>0$ one has

$\left(u_{i}+u_{i^{-}}\right) \Phi_{i}\left(u_{i}\right)=-\rho_{i}(t) \operatorname{sign}\left(S_{i}\right) \Phi_{i}\left(u_{i}\right) \geq m_{i-}^{*} \rho_{i}^{2}(t)\left[\operatorname{sign}\left(S_{i}\right)\right]^{2} \geq \eta \rho_{i}^{2}(t)$,

and for $S_{i}<0$ one has

$\left(u_{i}-u_{i+}\right) \Phi_{i}\left(u_{i}\right)=-\rho_{i}(t) \operatorname{sign}\left(S_{i}\right) \Phi_{i}\left(u_{i}\right) \geq m_{i+}^{*} \rho_{i}^{2}(t)\left[\operatorname{sign}\left(S_{i}\right)\right]^{2} \geq \eta \rho_{i}^{2}(t)$,

Then, for $S_{i}<0$ and $S_{i}>0$, one has

$-\rho_{i}(t) \operatorname{sign}\left(S_{i}\right) \Phi_{i}\left(u_{i}\right) \geq \eta \rho_{i}^{2}(t)$ 
Using (42) and the fact that $S_{i}^{2}>0$ and $S_{i} \operatorname{sign}\left(S_{i}\right)=\left|S_{i}\right|$ yields

$$
\begin{gathered}
-\rho_{i}(t) S_{i}^{2} \operatorname{sign}\left(S_{i}\right) \Phi_{i}\left(u_{i}\right) \geq \eta \rho_{i}^{2}(t) S_{i}^{2} \\
=\eta \rho_{i}^{2}(t)\left|S_{i}\right|^{2}
\end{gathered}
$$

Finally, because $\rho_{i}(t)>0$, thus for all $S_{i}$ one has

$$
S_{i} \Phi_{i}\left(u_{i}\right) \leq-\eta \rho_{i}(t)\left|S_{i}\right|
$$

And using the expressions (34), (35), (44), and (37), (39) becomes

$$
\begin{aligned}
\dot{V} & \leq \sum_{i=1}^{p}\left|S_{i}\right| k_{0 i}+\sum_{i=1}^{p}\left|S_{i}\right| \theta_{i}^{T} \psi_{i}(x)+\frac{1}{\eta} \sum_{i=1}^{p} S_{i} \Phi_{i}\left(u_{i}\right) \\
& -\sum_{i=1}^{p} \sigma_{0 i} \widetilde{k}_{0 i} k_{0 i}-\sum_{i=1}^{p} \sigma_{1 i} \widetilde{\theta}_{i}^{T} \theta_{i} \leq \sum_{i=1}^{p}\left|S_{i}\right| k_{0 i}+\sum_{i=1}^{p}\left|S_{i}\right| \theta_{i}^{T} \psi_{i}(x) \\
& +\sum_{i=1}^{p}-\rho_{i}(t)\left|S_{i}\right|-\sum_{i=1}^{p} \sigma_{0 i} \widetilde{k}_{0 i} k_{0 i}-\sum_{i=1}^{p} \sigma_{1 i} \widetilde{\theta}_{i}^{T} \theta_{i}=-\sum_{i=1}^{p} k_{1 i} S_{i}^{2} \\
& -\sum_{i=1}^{p} \sigma_{0 i} \widetilde{k}_{0 i} k_{0 i}-\sum_{i=1}^{p} \sigma_{1 i} \widetilde{\theta}_{i}^{T} \theta_{i}
\end{aligned}
$$

One can henceforth easily check that

$-\sigma_{0 i} \widetilde{k}_{0 i} k_{0 i} \leq-\frac{\sigma_{0 i}}{2} \widetilde{k}_{0 i}^{2}+\frac{\sigma_{0 i}}{2} k_{0 i}^{* 2}$

$-\sigma_{1 i} \widetilde{\theta}_{i}^{T} \theta_{i} \leq-\frac{\sigma_{1 i}}{2}\left\|\widetilde{\theta}_{i}\right\|^{2}+\frac{\sigma_{1 i}}{2}\left\|\theta_{i}^{*}\right\|^{2}$

And using the previous inequalities, (45) becomes

$$
\begin{aligned}
\dot{V} & \leq-\sum_{i=1}^{p} k_{1 i} S_{i}^{2}-\sum_{i=1}^{p} \frac{\sigma_{0 i}}{2} \widetilde{k}_{0 i}^{2}-\sum_{i=1}^{p} \frac{\sigma_{1 i}}{2}\left\|\tilde{\theta}_{i}\right\|^{2}+\sum_{i=1}^{p} \frac{\sigma_{0 i}}{2} k_{0 i}^{* 2} \\
& +\sum_{i=1}^{p} \frac{\sigma_{1 i}}{2}\left\|\theta_{i}^{*}\right\|^{2}
\end{aligned}
$$

Taking into account the Assumption 2, there exists a positive scalar $\sigma_{\text {go }}$ such that $G(x) \geq \sigma_{g 0} I_{p}$ and henceforth

$S^{T} G^{-1}(x) S=S^{T} G_{1}(x) S \leq \frac{1}{\sigma_{g 0}}\|S\|^{2}$

And using (46) and (47) yields

$\dot{V} \leq-\mu_{1} V+\pi_{1}$

with

$\pi_{1}=\sum_{i=1}^{p} \frac{\sigma_{0 i}}{2} k_{0 i}^{* 2}+\sum_{i=1}^{p} \frac{\sigma_{1 i}}{2}\left\|\theta_{i}^{*}\right\|^{2}$ where $\mu_{1}=\min \left\{\min _{i}\left\{2 \eta \sigma_{g 0} k_{1 i}\right\}, \min _{i}\left\{\gamma_{0 i} \sigma_{0 i}\right\}, \min _{i}\left\{\gamma_{1 i} \sigma_{1 i}\right\}\right\}$. Multiplying (48) by $e^{\mu_{1} t}$ yields

$\frac{d}{d t}\left(V e^{\mu_{1} t}\right) \leq \pi_{1} e^{\mu_{1} t}$

And integrating (49) over $[0, t]$, one has

$0 \leq V(t) \leq \frac{\pi_{1}}{\mu_{1}}+\left(V(0)-\frac{\pi_{1}}{\mu_{1}}\right) e^{-\mu_{1} t}$

Therefore, all variables of the control system, i.e., $k_{0 i}, \theta_{i}, S_{i}, E$ and $x$ are UUB. And hence the input $u_{i}$ is bounded.

With bearing in mind of (38), one can define $V(0)$ as follows

$$
\begin{aligned}
V(0)= & \frac{1}{2 \eta} S(0)^{T} G_{1}(x(0)) S(0)+\frac{1}{2} \sum_{i=1}^{p} \frac{1}{\gamma_{0 i}}\left(k_{0 i}(0)-k_{0 i}^{*}\right)^{2} \\
& +\frac{1}{2} \sum_{i=1}^{p} \frac{1}{\gamma_{1 i}}\left(\theta_{i}(0)-\theta_{i}^{*}\right)^{T}\left(\theta_{i}(0)-\theta_{i}^{*}\right)
\end{aligned}
$$

Since $G_{1}(x)$ is symmetric and positive definite, i.e., there exists an unknown positive constant $\sigma_{g 1}$ such that: $G_{1}(x) \geq \sigma_{g 1} I_{p}$, it follows from (50) and (38) that

$\left|S_{i}\right| \leq\left(\frac{2 \eta}{\sigma_{g 1}}\left(\frac{\pi_{1}}{\mu_{1}}+\left(V(0)-\frac{\pi_{1}}{\mu_{1}}\right) e^{-\mu_{1} t}\right)\right)^{1 / 2}$

Then, the solution of $S_{i}$ exponentially converges to a bounded adjustable domain defined as follows $\Omega_{S_{i}}=$ $\left\{S_{i}|| S_{i} \mid \leq\left(\frac{2 \eta}{\sigma_{g 1}} \frac{\pi_{1}}{\mu_{1}}\right)^{1 / 2}\right\}$. This ends the proof of the theorem.

Remark 2 Since the fuzzy approximation (30) is only guaranteed with a compact set, the proposed stability results in this paper are semiglobal in the sense that for any bounded initial states, there exists a fuzzy controller with sufficient large number of fuzzy rules such that the function approximation (30) holds for possible operating region.

Remark 3 According to the definition of $\mu_{1}$ and $\pi_{1}$, it can be seen that the size of $\mu_{1}$ depends on the controller design parameters $\gamma_{0 i}, \gamma_{1 i}, \sigma_{0 i}, \sigma_{1 i}$, and $k_{1 i}$ (which must be chosen strictly positive) and that of $\mu_{1}$ depends on the controller design parameters $\sigma_{0 i}$ and $\sigma_{1 i}$. It is very clear that if we increase $\gamma_{0 i}, \gamma_{1 i}$ and $k_{1 i}$ and decrease $\sigma_{0 i}$ and $\sigma_{1 i}$, it will help to reduce the term $\left(2 \eta \pi_{1} / \sigma_{g 1} \mu_{1}\right)^{1 / 2}$. This implies that the filtered tracking errors $S_{i}$ and the tracking error $E$ can be made arbitrary small by appropriately choosing those design parameters. 
3.2 The second state feedback fuzzy adaptive controller

The fuzzy adaptive controller presented above is only applicable for nonlinear systems with a symmetric and positive definite control-gain matrix, e.g., robotic systems and some electrical machines. Such a property is not satisfied for several physical systems, e.g., the visual serving and the automotive thermal management systems [32]. In the following, one presents a fuzzy adaptive controller in the case where the control-gain matrix is no longer symmetric and positive definite using a suitable matrix decomposition that has been already introduced in $[20,25,33-35]$.

\subsubsection{Decomposition of the control-gain matrix}

If the control-gain matrix is with non-zero leading principal minors [36], it can be always decomposed into the product of a symmetric positive definite matrix, a diagonal matrix whose elements are +1 or -1 and a unity upper triangular matrix as pointed out by the following result.

Lemma 1 Any real matrix $G(x) \in R^{p \times p}$ with non-zero leading principal minors can be decomposed as follows $[34,35]$ :

$G(x)=G_{s}(x) D T(x)$

where $G_{s}(x) \in R^{p \times p}$ is a symmetric positive definite matrix, $D \in R^{p \times p}$ is a diagonal matrix whose elements are +1 or -1 and $T(x) \in R^{p \times p}$ is a unity upper triangular matrix.

\section{Proof See [34].}

It is worth noting that the decomposition (52) of the matrix $G(x)$ is very useful. The symmetric positive definite matrix $G_{s}(x)$ will be particularly exploited in the stability analysis. The unity upper triangular matrix $T(x)$ allows for algebraic loop free sequential determination of the control components. The diagonal elements are nothing than the ratios of the signs of the leading principal minors of the control-gain matrix. This implies the following facts when the control-gain matrix has non-zero leading principal minors.

- If $G(x)$ is positive definite, then $D=I_{P}$,

- If $G(x)$ is negative definite, then $D=-I_{p}$, and

- If $G(x)$ is indefinite, the diagonal elements of the matrix $D$ are +1 and -1 .

\subsubsection{Fuzzy adaptive control design}

Consider the system (2) subject to Assumption 1 and the following assumption.
Assumption 4 The control-gain matrix is of class $C^{1}$, has non-zero leading principal minors of known signs and satisfies the following property

$\partial g_{i j}(x) / \partial y_{i}^{\left(r_{i}-1\right)}=0, \quad \forall i=1,2, \ldots, p$ and $j=1,2, \ldots, p$.

Using the matrix composition (52) and Eq. (20), the dynamics of $S$ can be rewritten as follows.

$G_{s}^{-1}(x) \dot{S}=G_{s}^{-1}(x)[v+F(x)]+D T(x) \Phi(u)$

with

$v=C_{r}^{T} E-y_{d}^{(r)}$.

Letting $G_{s 1}(x)=G_{s}^{-1}(x)$ and $F_{1}(x, u)=G_{s}^{-1}(x)[v+F(x)]+$ $[D T(x)-D] \Phi(u)$, Eq. (53) becomes

$G_{s 1}(x) \dot{S}=F_{1}(x, u)+D \Phi(u)$

Now, the following change of the variables for stability analysis and control design simplicity are introduced

$\bar{S}=D^{-1} S$

or $\bar{S}_{i}=d_{i i} S_{i}$ as $D=D^{T}=D^{-1}$ and $d_{i i}=+1$ or -1 .

This allows to rewrite (54) under the form

$G_{s 2}(x) \dot{\bar{S}}=D^{-1} F_{1}(x, u)+\Phi(u)$

where $G_{s 2}(x)=D^{-1} G_{s 1}(x) D$. Due to the special forms of the matrices $D$ and $G_{s 1}(x), G_{s 2}(x)$ preserves the important properties of the original matrix $G_{s 1}(x)$ (or $G_{s}(x)$ ). Indeed, one can easily show that $G_{s 2}(x)$ is also symmetric and positive definite. This property is of fundamental interest when investigating the control system stability.

Similarly to the first fuzzy adaptive control system, (56) can be rearranged as follows

$G_{s 2}(x) \dot{\bar{S}}+\frac{1}{2} \dot{G}_{s 2} \bar{S}=\alpha(\bar{z})+\Phi(u)$

where $\alpha(\bar{z})=\left[\alpha_{1}\left(\bar{z}_{1}\right), \alpha_{2}\left(\bar{z}_{2}\right), \ldots, \alpha_{p}\left(\bar{z}_{p}\right)\right]^{T}=D^{-1} F_{1}(x, u)+$ $\frac{1}{2} \dot{G}_{s 2}(x) \bar{S}$, with $\bar{z}=\left[\bar{z}_{1}^{T}, \bar{z}_{2}^{T}, \ldots, \bar{z}_{p}^{T}\right]^{T}$.

By carefully examining the expression of $F_{1}(x, u)$ and $\alpha(\bar{z})$, the elements of the vector $\bar{z}$ can be selected as follows:

$$
\begin{gathered}
\bar{z}_{1}=\left[x^{T}, S^{T}, u_{2}, \ldots, u_{p}\right]^{T} \\
\bar{z}_{2}=\left[x^{T}, S^{T}, u_{3}, \ldots, u_{p}\right]^{T} \\
\vdots \\
\bar{z}_{p-1}=\left[x^{T}, S^{T}, u_{P}\right]^{T} \\
\bar{z}_{p}=\left[x^{T}, S^{T}\right]^{T}
\end{gathered}
$$


It is clear from the propriety of the matrix of $D T(x)-D$ that $\bar{z}_{1}$ depends on control inputs $u_{2}, \ldots, u_{p}, \bar{z}_{2}$ depends on $u_{3}, \ldots, u_{p}$, and so on. The nonlinearities $\alpha(\bar{z})$ has an upper triangular control structure, allowing thereby for algebraic loop free sequential determination of the control variables.

Let us define the following compact sets

$$
\begin{gathered}
\Omega_{\bar{z}_{i}}=\left\{\left[x^{T}, S^{T}, u_{i+1}, \ldots, u_{p}\right]^{T} \mid x \in \Omega_{x} \subset R^{r}, x_{d} \in \Omega_{x_{d}}\right\} \\
\text { for } i=1,2, \ldots, p-1
\end{gathered}
$$

and

$\Omega_{\bar{z}_{p}}=\left\{\left[x^{T}, S^{T}\right] \mid x \in \Omega_{x} \subset R^{r}, x_{d} \in \Omega_{x_{d}}\right\}$

and introduce the following assumption.

Assumption 5 There exists an unknown continuous positive function $\bar{\alpha}_{i}\left(\bar{z}_{i}\right)$ such that

$\left|\alpha_{i}\left(\bar{z}_{i}\right)\right| \leq \eta \bar{\alpha}_{i}\left(\bar{z}_{i}\right), \quad \forall \bar{z}_{i} \in \Omega_{\bar{z}_{i}}$ where $\eta=\min _{i}\left\{\eta_{i}\right\}$.

The choice of the vectors $\bar{z}_{i}$, i.e., the input arguments of the unknown functions $\bar{\alpha}_{i}$, is not unique. Indeed, since $S$ and $u$ are functions of state $x$ and $x_{d}$, then it can be easily seen that all vectors $\bar{z}_{i}$ are functions of $x$ and $x_{d}$, e.g., one can choose $\bar{z}_{i}=\left[x^{T}, x_{d}{ }^{T}\right]^{T}$ or $\bar{z}_{i}=\left[x^{T}, E^{T}\right]^{T}$ for $i=1,2, \ldots, p$. Moreover, since $x_{d}$ is bounded, one can choose $\bar{z}_{i}=x$.

As in Section 3.1, the unknown continuous function $\bar{\alpha}_{i}\left(\bar{z}_{i}\right)$ can be approximated by a fuzzy system as follows

$\widehat{\bar{\alpha}}_{i}\left(\bar{z}_{i}, \theta\right)=\theta_{i}^{T} \psi_{i}\left(\bar{z}_{i}\right)$

where $\psi_{i}(\bar{z})_{i}$ is the FBF vector, which is fixed a priori by the designer, and $\theta_{i}$ is the adjustable parameter vector of the fuzzy system. Furthermore, the functions $\bar{\alpha}_{i}\left(\bar{z}_{i}\right)$ can be approximated optimally as follows:

$\bar{\alpha}_{i}\left(\bar{z}_{i}\right)=\hat{\bar{\alpha}}_{i}\left(\bar{z}_{i}, \theta_{i}^{*}\right)+\delta_{i}\left(\bar{z}_{i}\right)=\theta_{i}^{* T} \psi_{i}\left(\bar{z}_{i}\right)+\delta_{i}\left(\bar{z}_{i}\right)$

and the fuzzy approximation error is assumed to be bounded as usual $[1,26,27]$, i.e.,

$\left|\delta_{i}\left(\bar{z}_{i}\right)\right| \leq \bar{\delta}_{i}, \quad \forall \bar{z}_{i} \in \Omega_{\bar{z}_{i}}$

where $\bar{\delta}_{i}$ is an unknown constant.
From the above analysis, one has

$$
\begin{aligned}
\widehat{\bar{\alpha}}_{i}\left(\bar{z}_{i}, \theta_{i}\right)-\bar{\alpha}_{i}\left(\bar{z}_{i}\right) & =\widehat{\bar{\alpha}}_{i}\left(\bar{z}_{i}, \theta_{i}\right)-\widehat{\bar{\alpha}}_{i}\left(\bar{z} i, \theta_{i}^{*}\right)+\widehat{\bar{\alpha}}_{i}\left(\bar{z}_{i}, \theta_{i}^{*}\right)-\bar{\alpha}_{i}\left(\bar{z}_{i}\right), \\
& =\hat{\bar{\alpha}}_{i}\left(\bar{z}_{i}, \theta_{i}\right)-\hat{\bar{\alpha}}_{i}\left(\bar{z}_{i}, \theta_{i}^{*}\right)-\delta_{i}\left(\bar{z}_{i}\right), \\
& =\tilde{\theta}_{i}^{T} \psi_{i}\left(\bar{z}_{i}\right)-\delta_{i}\left(\bar{z}_{i}\right)
\end{aligned}
$$

The following fuzzy adaptive variable-structure controller is proposed to perform the required tracking control objective:

$u_{i}= \begin{cases}-\bar{\rho}_{i}(t) \operatorname{sign}\left(\bar{S}_{i}\right)-u_{i^{-}}, & \bar{S}_{i}>0, \\ 0, & \bar{S}_{i}=0, \\ -\bar{\rho}_{i}(t) \operatorname{sign}\left(\bar{S}_{i}\right)+u_{i+}, & \bar{S}_{i}<0,\end{cases}$

with

$$
\begin{gathered}
\bar{\rho}_{i}(t)=k_{0 i}+k_{1 i}\left|\bar{S}_{i}\right|+\theta_{i}^{T} \psi_{i}\left(\bar{z}_{i}\right) \quad \forall i=1, \ldots, p \\
\dot{k}_{0 i}=-\gamma_{0 i} \sigma_{0 i} k_{0 i}+\gamma_{0 i}\left|\bar{S}_{i}\right|, \text { with } k_{0 i}(0)>0 \\
\dot{\theta}_{i}=-\gamma_{1 i} \sigma_{1 i} \theta_{i}+\gamma_{1 i}\left|\bar{S}_{i}\right| \psi_{i}\left(\bar{z}_{i}\right), \text { with } \theta_{i j}(0)>0
\end{gathered}
$$

where $\gamma_{0 i}, \gamma_{1 i}, \sigma_{0 i}, \sigma_{1 i}, k_{1 i}>0$ are design constants, and $k_{0 i}$ and $\theta_{i}$ are the online estimates of the uncertain terms $k_{0 i}^{*}=\bar{\delta}_{i}$ and $\theta_{i}^{*}$, respectively.

Multiplying (57) by $\bar{S}^{T}$ and using Assumption 5 yields

$$
\begin{aligned}
\frac{1}{2 \eta} \bar{S}^{T} \dot{G}_{s 2}(x) \bar{S} & +\frac{1}{\eta} \bar{S}^{T} G_{s 2}(x) \dot{\bar{S}}=\frac{1}{\eta} \bar{S}^{T} \alpha(\bar{z})+\frac{1}{\eta} \bar{S}^{T} \Phi(u) \\
& \leq \sum_{i=1}^{p}\left|\bar{S}_{i}\right| \bar{\alpha}_{i}\left(\bar{z}_{i}\right)+\frac{1}{\eta} \bar{S}^{T} \Phi(u)
\end{aligned}
$$

And taking into account (61) and (65), one has

$$
\begin{aligned}
\frac{1}{2 \eta} \bar{S}^{T} \dot{G}_{s 2}(x) \bar{S} & +\frac{1}{\eta} \bar{S}^{T} G_{s 2}(x) \dot{\bar{S}} \leq \sum_{i=1}^{p}\left|\bar{S}_{i}\right| \bar{\alpha}_{i}\left(\bar{z}_{i}\right)+\frac{1}{\eta} \bar{S}^{T} \Phi(u) \\
& \leq-\sum_{i=1}^{p}\left|\bar{S}_{i}\right|\left(\widetilde{k}_{0 i}+\widetilde{\theta}_{i}^{T} \psi_{i}\left(\bar{z}_{i}\right)\right) \\
& +\sum_{i=1}^{p}\left|\bar{S}_{i}\right|\left(k_{0 i}+\theta_{i}^{T} \psi_{i}\left(\bar{z}_{i}\right)\right)+\frac{1}{\eta} \bar{S}^{T} \Phi(u)
\end{aligned}
$$

where $\widetilde{\theta}_{i}=\theta_{i}-\theta_{i}^{*}$ and $\widetilde{k}_{0 i}=k_{0 i}-k_{0 i}^{*}=k_{0 i}-\bar{\delta}_{i}$.

Theorem 2 Consider the system (2) subject to Assumptions 1 , 4 and 5. Then, the control law defined by Eqs. (62) to (64) ensures the following properties:

- All the variables in the closed-loop system are UUB. 
- The filtered tracking errors $S_{i}$ of the control system exponentially converge to an adjustable domain defined as:

$$
\Omega_{S_{i}}=\left\{S_{i}|| S_{i} \mid \leq\left(\frac{2 \eta}{\sigma_{g 2}} \frac{\pi_{2}}{\mu_{2}}\right)^{1 / 2}\right\}
$$

where $\sigma_{g 2}$ satisfies $\sigma_{g 2} I_{p} \leq G_{s 2}(x), \pi_{2}=\pi_{1}$, and

$\mu_{2}=\min \left\{\min _{i}\left\{2 \eta \sigma_{g 2} k_{1 i}\right\}, \min _{i}\left\{\gamma_{0 i} \sigma_{0 i}\right\}, \min _{i}\left\{\gamma_{1 i} \sigma_{1 i}\right\}\right\}$.

Proof It is carried out using the following Lyapunov function candidate

$V=\frac{1}{2 \eta} \bar{S}^{T} G_{S 2}(x) \bar{S}+\frac{1}{2} \sum_{i=1}^{p} \frac{1}{\gamma_{1 i}} \widetilde{\theta}_{i}^{T} \tilde{\theta}_{i}+\frac{1}{2} \sum_{i=1}^{p} \frac{1}{\gamma_{0 i}} \widetilde{k}_{i}^{2}$.

Since this proof is very similar to that of Theorem 1 , it is omitted.

\section{Design of the output feedback fuzzy adaptive controller}

In previous section, fuzzy adaptive variable structure controllers have been developed for multivariable systems with actuators nonlinearities using the state feedback, based on the assumption that all the states system are measurable. It may be impossible in reality to measure all the system states $[37,38]$. In the case where only the output vector $y=\left[y_{1}, \ldots, y_{p}\right]^{T}$ is available, an observer is needed to estimate $y_{i}^{(j)}$, for $j=$ $1, \ldots, r_{i}-1$. Below, a high-gain observer [39-41] is used to reconstruct the missing states of the system.

Lemma 2 Suppose the function $y_{i}(t)$ and its first derivatives $r_{i}$ are bounded. Consider the following linear system, for $i=1, \ldots, p$ :

$$
\left\{\begin{array}{l}
\varepsilon \dot{\pi}_{i 1}=\pi_{i 2} \\
\varepsilon \dot{\pi}_{i 2}=\pi_{i 3} \\
\vdots \\
\varepsilon \dot{\pi}_{i r_{i}}=-b_{i 1} \pi_{i r_{i}}-\cdots-b_{i\left(r_{i}-1\right)} \pi_{i 2}-\pi_{i 1}+y_{i}
\end{array}\right.
$$

Where $\varepsilon$ is any small positive constant, the parameters $b_{i 1}, \cdots, b_{i\left(r_{i}-1\right)}$ are chosen so that the polynomial $s^{r_{i}}+b_{i 1} s^{\left(r_{i}-1\right)}+\cdots+b_{i\left(r_{i}-1\right)} s+1 \quad$ is Hurwitz. Then, there exists positive constants $h_{i k}, k=2, \ldots, r_{i}$ and for $i=1, \ldots, p$, and $t^{*}$ such that for all $t>t^{*}$ we have:

$$
\begin{aligned}
& \frac{\pi_{i(k+1)}}{\varepsilon^{k}}-y_{i}^{(k)}=-\varepsilon w_{i}^{(k+1)}, \quad k=1, \ldots, r_{i}-1 \\
& \left|\frac{\pi_{i(k+1)}}{\varepsilon^{k}}-y_{i}^{(k)}\right| \leq \varepsilon h_{i(k+1)}, \quad k=1, \ldots, r_{i}-1
\end{aligned}
$$

where $w_{i}=\pi_{i r_{i}}+b_{i 1} \pi_{i\left(r_{i}-1\right)}+\cdots+b_{i\left(r_{i}-1\right)} \pi_{i 1}, w_{i}^{(k)}$ denotes the $k$ th derivative of $w_{i}$, and $\left|w_{i}^{(k)}\right| \leq h_{i k}$.

Proof The proof of this lemma can be found in [41, 42].

Now, let us consider the system (2) subject to Assumptions 1,4 , and 5 . Having the observers $(i=1, \ldots, p)$ in $(68)$, we can define the following variables:

$$
\begin{aligned}
& W_{i}=\left[0, w_{i}^{(2)}, \ldots, w_{i}^{\left(r_{i}\right)}\right]^{T}, \quad i=1, \ldots, p, W=\left[W_{1}^{T}, W_{2}^{T}, \ldots, W_{p}^{T}\right]^{T}, \\
& \pi_{i}=\left[\pi_{i 1}, \pi_{i 2}, \ldots, \pi_{i r_{i}}\right]^{T}, \quad i=1, \ldots, p, \pi=\left[\pi_{1}^{T}, \pi_{2}^{T}, \ldots, \pi_{p}^{T}\right]^{T}, \\
& \widehat{x}_{i}=\left[x_{i 1}, \frac{\pi_{i 2}}{\varepsilon}, \frac{\pi_{i 3}}{\varepsilon^{2}}, \ldots, \frac{\pi_{i r_{i}}}{\varepsilon^{r_{i}-1}}\right]^{T}, i=1, \ldots, p, \widehat{x}=\left[\widehat{x}_{1}^{T}, \widehat{x}_{2}^{T}, \ldots, \widehat{x}_{p}^{T}\right]^{T}, \\
& x_{d i}=\left[y_{d i}, \dot{y}_{d i}, \ldots, y_{d i}^{\left(r_{i}-1\right)}\right]^{T}, \quad i=1, \ldots, p, x_{d}=\left[x_{d 1}^{T}, x_{d 2}^{T}, \ldots, x_{d p}^{T}\right]^{T} \text {, } \\
& \widehat{S}=\left[\begin{array}{c}
\widehat{S}_{1} \\
\vdots \\
\widehat{S}_{p}
\end{array}\right]=\left[\begin{array}{c}
C_{1}^{T}\left(\widehat{x}_{1}-x_{d 1}\right) \\
\vdots \\
C_{p}^{T}\left(\widehat{x}_{p}-x_{d p}\right)
\end{array}\right],
\end{aligned}
$$

Now, we can show as in $[39,40]$ that $\widehat{S}$ and its dynamics can be given by:

$\widehat{S}=S-\varepsilon\left[\begin{array}{c}C_{1}^{T} W_{1} \\ \vdots \\ C_{p}^{T} W_{p}\end{array}\right]=S-\varepsilon C^{T} W$

$\dot{\widehat{S}}=\dot{S}-\varepsilon C^{T} \dot{W}$

The vectors $C_{1}, \ldots, C_{p}$ have been previously defined in section 2 .

From (20), (52), and (72), the dynamics of $\widehat{S}$ can be rewritten as follows.

$G_{s}^{-1}(x) \dot{\hat{S}}=G_{s}^{-1}(x)[v+F(x)]+D T(x) \Phi(u)-\varepsilon G_{s}^{-1}(x) C^{T} \dot{W}$

with $v=C_{r}^{T} E-y_{d}^{(r)}$. Letting $G_{s 1}(x)=G_{s}^{-1}(x)$ and $F_{1}(x, u)=G_{s}^{-1}(x)[v+F(x)]+[D T(x)-D] \Phi(u)$, Eq. (73) becomes

$G_{s 1}(x) \dot{\widehat{S}}=F_{1}(x, u)+D \Phi(u)-\varepsilon G_{s 1}(x) C^{T} \dot{W}$

Now, we use the following change of coordinates

$\widehat{\bar{S}}=D^{-1} \widehat{S}$

Recall that $D=D^{T}=D^{-1}$. This allows to rewrite (74) as

$G_{s 2}(x) \dot{\hat{S}}=D^{-1} F_{1}(x, u)+\Phi(u)-\varepsilon G_{s 2}(x) D^{-1} C^{T} \dot{W}$

With $G_{s 2}(x)=D^{-1} G_{s 1}(x) D$. 
Similar to the previous section, (76) can be arranged as follows

$G_{s 2}(x) \dot{\widehat{S}}+\frac{1}{2} \dot{G}_{s 2} \widehat{\bar{S}}=\alpha(\bar{z})+\Phi(u)-\varepsilon G_{s 2}(x) D^{-1} C^{T} \dot{W}$

where $\alpha(\bar{z})=\left[\alpha_{1}\left(\bar{z}_{1}\right), \alpha_{2}\left(\bar{z}_{2}\right), \ldots, \alpha_{p}\left(\bar{z}_{p}\right)\right]^{T}=D^{-1} F_{1}(x, u)+$ $\frac{1}{2} \dot{G}_{s 2}(x) \hat{\bar{S}}$, with $\bar{z}=\left[\bar{z}_{1}^{T}, \bar{z}_{2}^{T}, \ldots, \bar{z}_{p}^{T}\right]^{T}$. Recall that the nonlinearities $\alpha(\bar{z})$ has an upper triangular control structure, allowing thereby for algebraic loop free sequential determination of the control variables. Then, the inputs $\bar{z}_{1}$ can be selected as in the previous section. The functions $\alpha_{i}\left(\bar{z}_{i}\right)$ are assumed to satisfy Assumption 5.

As in the previous section, the unknown continuous functions $\alpha_{i}\left(\bar{z}_{i}\right)$ (for $i=1, \ldots, p$ ) can be approximated by adaptive fuzzy systems as follows

$\widehat{\bar{\alpha}}_{i}\left(\widehat{\bar{z}}_{i}, \theta\right)=\theta_{i}^{T} \psi_{i}\left(\widehat{\bar{z}}_{i}\right)$

where $\widehat{\bar{z}}_{i}$ is an estimate of $\bar{z}_{i}$. The elements of $\widehat{\bar{z}}_{i}$ can be directly determined from the designed observers (68).

Similarly to the previous section, we have

$$
\begin{aligned}
& \bar{\alpha}_{i}\left(\bar{z}_{i}\right)=\theta_{i}^{T} \psi_{i}\left(\widehat{\bar{z}}_{i}\right)-\tilde{\theta}_{i}^{T} \psi_{i}\left(\widehat{\bar{z}}_{i}\right)+\delta_{i}\left(\bar{z}_{i}\right) \\
& +\theta_{i}^{* T}\left[\psi_{i}\left(\widehat{\bar{z}}_{i}\right)-\psi_{i}\left(\bar{z}_{i}\right)\right]
\end{aligned}
$$

This fuzzy approximation error $\delta_{i}\left(\bar{z}_{i}\right)+\theta_{i}^{* T}\left[\psi_{i}\left(\overline{\bar{z}}_{i}\right)-\psi_{i}\left(\bar{z}_{i}\right)\right]$ can be assumed to be bounded as usual $[1,26,27]$, i.e., one has

$\left|\delta_{i}\left(\bar{z}_{i}\right)+\theta_{i}^{* T}\left[\psi_{i}\left(\widehat{\bar{z}}_{i}\right)-\psi_{i}\left(\bar{z}_{i}\right)\right]\right| \leq \bar{\delta}_{i}, \quad \forall \bar{z}_{i} \in \Omega$

Let us introduce the following assumption

\section{Assumption 6 We assume}

$\frac{\varepsilon}{\eta} \widehat{\bar{S}}^{T} G_{S 2}(x) D^{-1} C^{T} \dot{W} \leq \varepsilon \kappa_{1}\|\widehat{\bar{S}}\|^{2}+\varepsilon \kappa_{2}$

where $\kappa_{1}$ and $\kappa_{2}$ are unknown constants.

Remark 4 Note that Assumption 6 is not restrictive for the following reasons:

- $\dot{W}$ is generally assumed to be bounded, please see [39-42].

- $G_{s 1}(x)$ is a symmetric positive-definite matrix, i.e., $\exists \sigma_{g 1}$ $>0$ such as $G_{s 1}(x) \geq \sigma_{g 1} I$. Then, $\widehat{\bar{S}}^{T} G_{s 2}(x) \widehat{\bar{S}} \leq \frac{1}{\sigma_{g 1}}\|\widehat{\bar{S}}\|^{2}$.
The following fuzzy adaptive variable-structure controller is proposed to achieve the control objective:

$u_{i}= \begin{cases}-\hat{\bar{\rho}}_{i}(t) \operatorname{sign}\left(\hat{\bar{S}}_{i}\right)-u_{i-}, & \widehat{\bar{S}}_{i}>0, \\ 0, & \widehat{\bar{S}}_{i}=0, \\ -\widehat{\bar{\rho}}_{i}(t) \operatorname{sign}\left(\hat{\bar{S}}_{i}\right)+u_{i+}, & \overline{\bar{S}}_{i}<0,\end{cases}$

with

$$
\begin{gathered}
\widehat{\bar{\rho}}_{i}(t)=k_{0 i}+k_{1 i}\left|\widehat{\widehat{S}}_{i}\right|+\theta_{i}^{T} \psi_{i}\left(\widehat{\bar{z}}_{i}\right), \quad \forall i=1, \ldots, p \\
\dot{k}_{0 i}=-\gamma_{0 i} \sigma_{0 i} k_{0 i}+\gamma_{0 i}\left|\hat{\bar{S}}_{i}\right|, \quad k_{0 i}(0)>0
\end{gathered}
$$

$\dot{\theta}_{i}=-\gamma_{1 i} \sigma_{1 i} \theta_{i}+\gamma_{1 i}\left|\widehat{\bar{S}}_{i}\right| \psi_{i}\left(\widehat{\bar{z}}_{i}\right)$, with $\theta_{i j}(0)>0$

where $\gamma_{0 i}, \gamma_{1 i}, \sigma_{0 i}, \sigma_{1 i}, k_{1 i}>\varepsilon \kappa_{1}$ are design constants, and $k_{0 i}$ and $\theta_{i}$ are the online estimates of the uncertain terms $k_{0 i}^{*}=\bar{\delta}_{i}$ and $\theta_{i}^{*}$, respectively.

Multiplying (77) by $\hat{\bar{S}}^{T}$ and using Assumptions 5 and 6 yields

$$
\begin{aligned}
& \frac{1}{2 \eta} \hat{\bar{S}}^{T} \dot{G}_{s 2}(x) \widehat{\bar{S}}+\frac{1}{\eta} \widehat{\bar{S}}^{T} G_{s 2}(x) \dot{\bar{S}}=\frac{1}{\eta} \widehat{\bar{S}}^{T} \alpha(\bar{z})+\frac{1}{\eta} \widehat{\widehat{S}}^{T} \Phi(u)-\frac{\varepsilon}{\eta} \widehat{\bar{S}}^{T} G_{s 2}(x)\left(D^{-1} C^{T} \dot{W}\right) \\
& \leq \sum_{i=1}^{p}\left|\widehat{S}_{i}\right| \bar{\alpha}_{i}\left(\bar{z}_{i}\right)+\frac{1}{\eta} \widehat{\widehat{S}}^{T} \Phi(u)+\varepsilon \kappa_{1}\|\hat{\widehat{S}}\|^{2}+\varepsilon \kappa_{2}
\end{aligned}
$$

from (79) and (85), one has

$$
\begin{aligned}
& \frac{1}{2 \eta} \widehat{\bar{S}}^{T} \dot{G}_{s 2}(x) \widehat{\bar{S}}+\frac{1}{\eta} \widehat{\bar{S}}^{T} G_{s 2}(x) \dot{\overline{\hat{S}}} \leq-\sum_{i=1}^{p}\left|\widehat{\bar{S}}_{i}\right|\left(\widetilde{k}_{0 i}+\tilde{\theta}_{i}^{T} \psi_{i}\left(\widehat{\bar{z}}_{i}\right)\right) \\
& +\sum_{i=1}^{p}\left|\widehat{\bar{S}}_{i}\right|\left(k_{0 i}+\theta_{i}^{T} \psi_{i}\left(\hat{\bar{z}}_{i}\right)\right)+\frac{1}{\eta} \widehat{\bar{S}}^{T} \Phi(u)+\varepsilon \kappa_{1}\|\hat{\bar{S}}\|^{2} \\
& +\varepsilon \kappa_{2}
\end{aligned}
$$

where $\widetilde{\theta}_{i}=\theta_{i}-\theta_{i}^{*}$ and $\widetilde{k}_{0 i}=k_{0 i}-k_{0 i}^{*}=k_{0 i}-\bar{\delta}_{i}$.

Theorem 3 Consider the system (2) with observers (68) and subject to Assumptions 1 and 4-6. Then, the control law defined by Eqs. (82) to (84) ensures the following properties: 
- All the variables in the closed-loop system are UUB.

- The filtered tracking error $S_{i}$ converges to an adjustable domain which can be defined by:

$$
\Omega_{S_{i}}=\left\{S_{i}|| S_{i} \mid \leq\left(\frac{2 \eta}{\sigma_{g 2}} \frac{\pi_{3}}{\mu_{3}}\right)^{1 / 2}+\varepsilon \bar{W}\right\}
$$

where $\sigma_{g 2}$ satisfies $\sigma_{g 2} I_{p} \geq G_{s 2}(x), \bar{W} \geq\left\|C^{T} W\right\|$, $\pi_{3}=\sum_{i=1}^{p} \frac{\sigma_{0 i}}{2} k_{0 i}^{* 2}+\sum_{i=1}^{p} \frac{\sigma_{1 i}}{2}\left\|\theta_{i}^{*}\right\|^{2}+\varepsilon \kappa_{2} \quad$ and $\quad \mu_{3}=\min$ $\left\{\min _{i}\left\{2 \eta \sigma_{g 2}\left(k_{1 i}-\varepsilon \kappa_{1}\right)\right\}, \min _{i}\left\{\gamma_{0 i} \sigma_{0 i}\right\}, \min _{i}\left\{\gamma_{1 i} \sigma_{1 i}\right\}\right\}$.

Proof It is carried out using the following Lyapunov function candidate

$V=\frac{1}{2 \eta} \widehat{\bar{S}}^{T} G_{s 2}(x) \widehat{\bar{S}}+\frac{1}{2} \sum_{i=1}^{p} \frac{1}{\gamma_{1 i}} \widetilde{\theta}_{i}^{T} \widetilde{\theta}_{i}+\frac{1}{2} \sum_{i=1}^{p} \frac{1}{\gamma_{0 i}} \widetilde{k}_{i}^{2}$

Since this proof is very similar to that of Theorem 1, it is omitted.

Remark 5 Two notes are worth to be made. Firstly, in the case where $u_{i+}=u_{i-}=u_{i 0}$, the expressions (33), (62), and (82) are respectively simplified as

$u_{i}=-\left(\rho_{i}(t)+u_{i 0}\right) \operatorname{sign}\left(S_{i}\right)$

$u_{i}=-\left(\bar{\rho}_{i}(t)+u_{i 0}\right) \operatorname{sign}\left(\bar{S}_{i}\right)$

$u_{i}=-\left(\widehat{\bar{\rho}}_{i}(t)+u_{i 0}\right) \operatorname{sign}\left(\widehat{\bar{S}}_{i}\right)$

where

$\rho_{i}(t)=k_{0 i}+k_{1 i}\left|S_{i}\right|+\theta_{i}^{T} \psi_{i}(x)$,

$\bar{\rho}_{i}(t)=k_{0 i}+k_{1 i}\left|\bar{S}_{i}\right|+\theta_{i}^{T} \psi_{i}\left(\bar{z}_{i}\right)$ and

$\widehat{\bar{\rho}}_{i}(t)=k_{0 i}+k_{1 i}\left|\widehat{\bar{S}}_{i}\right|+\theta_{i}^{T} \psi_{i}\left(\widehat{\bar{z}}_{i}\right)$.

Secondly, the sign function has to be replaced by any equivalent smooth function to deal with the chattering effects [43-45].

Remark 6 We can show without any technical difficulty that the proposed controller remains applicable for MIMO system (2) free of the input nonlinearities (i.e., in the case where $u_{i+}=$ $u_{i-}=0$, and $\left.\phi_{i+}\left(u_{i}\right)=\phi_{i-}\left(u_{i}\right)=1\right)$.
Remark 7 In the proposed adaptive laws, the $\sigma$-modification technique is used to prevent parameter drift. Also, we can use a projective operator method (1), and $e$ - modification method in place of $\sigma$-modification technique.

Remark 8 Table 1 summarizes the comparison between the three fuzzy adaptive controllers proposed in the paper:

Remark 9 Although the class of systems considered in our paper and that in [13] are all completely different, a simple theoretical comparison is made here and it is summarized in Table 2.

\section{Simulation results}

Simulation studies are carried out to show the effectiveness of the proposed adaptive fuzzy controllers. Two control problems are considered to this end. The first one concerns a twolink rigid robot manipulator (its model has naturally a symmetric control-gain matrix), while the second one concerns an academic multivariable nonlinear system (its model has a nonsymmetric and indefinite control-gain matrix).

5.1 Test of the fuzzy adaptive state feedback controllers

\subsubsection{Example 1 (Test of the first controller)}

In the following, one presents simulation results showing the performances of the first controller applied to a two-link rigid

Table 1 Comparison between the three proposed controllers

\begin{tabular}{|c|c|c|}
\hline & $\begin{array}{l}\text { Type of the } \\
\text { controller }\end{array}$ & Assumptions made \\
\hline Controller 1 & $\begin{array}{l}\text { A state feedback } \\
\text { controller }\end{array}$ & $\begin{array}{l}\text { The matrix } G(x) \text { is symmetric and } \\
\text { positive definite } \\
\text { The state vector is available for } \\
\text { measurement. }\end{array}$ \\
\hline Controller 2 & $\begin{array}{l}\text { A state feedback } \\
\text { controller }\end{array}$ & $\begin{array}{l}\text { The matrix } G(x) \text { is not necessary } \\
\text { symmetric and but with non-zero } \\
\text { leading principal minors. Their } \\
\text { signs are assumed to be known. } \\
\text { The state vector is available for } \\
\text { measurement. }\end{array}$ \\
\hline Controller 3 & $\begin{array}{l}\text { An output feedback } \\
\text { controller }\end{array}$ & $\begin{array}{l}\text { The matrix } G(x) \text { is not necessary } \\
\text { symmetric and but with non-zero } \\
\text { leading principal minors. Their } \\
\text { signs are assumed to be known. } \\
\text { The state vector is not measurable, } \\
\text { except the output vector. }\end{array}$ \\
\hline Remarks & $\begin{array}{l}\text { The class considered } \\
\text { of the second and } \\
\text { considered in the f } \\
\text { The third controller is } \\
\text { the second controll }\end{array}$ & $\begin{array}{l}\text { of the MIMO systems in the design } \\
\text { third controllers is large that the one } \\
\text { irst design. } \\
\text { s an output feedback extension of } \\
\text { ler. }\end{array}$ \\
\hline
\end{tabular}


Table 2 Comparison between our third controller and that proposed in [13]

\begin{tabular}{|c|c|c|}
\hline Comparison & Controller 3 proposed in this paper & Controller proposed in [13] \\
\hline $\begin{array}{l}\text { The class of MIMO } \\
\text { nonlinear system } \\
\text { considered }\end{array}$ & $y^{(r)}=F(x)+G(x) \Phi(u)$ & $\begin{array}{l}\dot{x}=A x+B(\Phi(u)+d(x, p, t)) \text { with } A^{n \times n} \text { is a state matrix, } B^{n \times p} \text { is a constant } \\
\quad \text { control gain matrix, } d(x, p, t) \in R^{p} \text { is the lumped uncertainties and external } \\
\text { disturbances, and } p(t) \text { is a vector of uncertain parameters. }\end{array}$ \\
\hline $\begin{array}{l}\text { Type of the controller } \\
\text { designed }\end{array}$ & $\begin{array}{l}\text { An output feedback fuzzy adaptive } \\
\text { variable structure controller }\end{array}$ & A state-feedback sliding mode controller \\
\hline $\begin{array}{l}\text { The control problem } \\
\text { considered }\end{array}$ & A tracking problem & A stabilization problem \\
\hline $\begin{array}{l}\text { Type of input } \\
\text { nonlinearities }\end{array}$ & Sector nonlinearities with dead zones & Sector nonlinearities with dead zones \\
\hline \multirow[t]{4}{*}{ Assumptions made } & The state vector is not measurable & The state vector is available for measurement \\
\hline & $F(x)$ and $G(x)$ are unknown & The matrices $A$ and $B$ are perfectly known. \\
\hline & The gain reduction tolerances of the & The gain reduction tolerances of the nonlinear dead-zones are known. \\
\hline & wn. & The nonlinear term $d(x, p, t)$ is bounded by a known function. \\
\hline Control gain matrix & $\begin{array}{l}\text { The control gain matrix } G(x) \text { is a } \\
\text { function the state vector. }\end{array}$ & $\begin{array}{l}\text { The control gain matrix } B \text { is constant. Remark. This assumption can be } \\
\text { considerably simplifying the controller design. }\end{array}$ \\
\hline $\begin{array}{l}\text { Singularity of the } \\
\text { controller }\end{array}$ & $\begin{array}{l}\text { Our controller is free of the singularity } \\
\text { problem. }\end{array}$ & The proposed controller in [13] can be singular, when $\|S\|=0$. \\
\hline Conclusions & \multicolumn{2}{|c|}{$\begin{array}{l}\text { Note that the class of the MIMO systems considered in [13] is simple and its model is almost known. Moreover, the } \\
\text { controller designed is a state-feedback and can be singular when }\|S\|=0 \text {. }\end{array}$} \\
\hline
\end{tabular}

robot manipulator which moves in a horizontal plane. The dynamic equations of this MIMO system are given by $[6,20$, 25]:

$$
\left(\begin{array}{l}
\ddot{q}_{1} \\
\ddot{q}_{2}
\end{array}\right)=\left(\begin{array}{ll}
M_{11} & M_{12} \\
M_{21} & M_{22}
\end{array}\right)^{-1}\left\{\left(\begin{array}{l}
\Phi_{1}\left(u_{1}\right) \\
\Phi_{2}\left(u_{2}\right)
\end{array}\right)-\left(\begin{array}{cc}
-h \dot{q}_{2} & -h\left(\dot{q}_{1}+\dot{q}_{2}\right) \\
h \dot{q}_{1} & 0
\end{array}\right)\left(\begin{array}{l}
\dot{q}_{1} \\
\dot{q}_{2}
\end{array}\right)\right\},
$$

where

$M_{11}=a_{1}+2 a_{3} \cos \left(q_{2}\right)+2 a_{4} \sin \left(q_{2}\right), M_{22}=a_{2}$,

$M_{21}=M_{12}=a_{2}+a_{3} \cos \left(q_{2}\right)+a_{4} \sin \left(q_{2}\right), h=a_{3} \cos \left(q_{2}\right)-a_{4} \cos \left(q_{2}\right)$,

with

$a_{1}=I_{1}+m_{1} l_{c 1}^{2}+I_{e}+m_{e} l_{c e}^{2}+m_{e} l_{1}^{2}, \quad a_{2}=I_{e}+m_{e} l_{c e}^{2}$,

$a_{3}=m_{e} l_{1} l_{c e} \cos \left(\delta_{e}\right), a_{4}=m_{e} l_{1} l_{c e} \sin \left(\delta_{e}\right)$.

Note that $\Phi_{i}\left(u_{i}\right)$, for $i=1,2$, are the considered input nonlinearities. The robot parameters are:

$m_{1}=1, m_{e}=2, l_{1}=1, l_{c 1}=0.5, l_{c e}=0.6, I_{1}=0.12$,

$I_{e}=0.25, \quad \delta_{e}=30^{\circ}$.

Let $\left[y_{1}, y_{2}\right]=\left[q_{1}, q_{2}\right], u=\left[u_{1}, u_{2}\right]^{T}, \Phi(u)=\left[\Phi_{1}\left(u_{1}\right), \Phi_{2}\right.$ $\left.\left(u_{2}\right)\right]^{T}, x=\left[q_{1}, \dot{q}_{1}, q_{2}, \dot{q}_{2}\right]^{T}$. One can rewrite the robot model with actuator nonlinearities (Eq. 92) as follows

$\ddot{y}=F(x)+G(x) \Phi(u)$ where

$$
\begin{gathered}
G(x)=\left(\begin{array}{ll}
g_{11}(x) & g_{12}(x) \\
g_{21}(x) & g_{22}(x)
\end{array}\right)=M^{-1}=\left(\begin{array}{ll}
M_{11} & M_{12} \\
M_{21} & M_{22}
\end{array}\right)^{-1} . \\
F(x)=\left(\begin{array}{l}
f_{1}(x) \\
f_{2}(x)
\end{array}\right)=-M^{-1}\left(\begin{array}{cc}
-h \dot{q}_{2} & -h\left(\dot{q}_{1}+\dot{q}_{2}\right) \\
h \dot{q}_{1} & 0
\end{array}\right)\left(\begin{array}{l}
\dot{q}_{1} \\
\dot{q}_{2}
\end{array}\right),
\end{gathered}
$$

The input nonlinearities are described as in [16]:

$$
\begin{aligned}
& \Phi_{1}\left(u_{1}\right)= \begin{cases}\left(u_{1}-3\right)\left(1.5-0.3 e^{0.3\left|\sin \left(u_{1}\right)\right|}\right), & u_{1}>3, \\
0, & \left|u_{1}\right| \leq 3, \\
\left(u_{1}+3\right)\left(1.5-0.3 e^{0.3\left|\sin \left(u_{1}\right)\right|}\right), & u_{1}<-3,\end{cases} \\
& \Phi_{2}\left(u_{2}\right)= \begin{cases}\left(u_{2}-3\right)\left(1.3-0.2 e^{0.1\left|\cos \left(u_{2}\right)\right|}\right), & u_{2}>3, \\
0, & \left|u_{2}\right| \leq 3, \\
\left(u_{2}+3\right)\left(1.3-0.2 e^{0.1\left|\cos \left(u_{2}\right)\right|}\right), & u_{2}<-3,\end{cases}
\end{aligned}
$$

The control objective consists in allowing the system outputs $q_{1}$ and $q_{2}$ to respectively track the sinusoidal-desired trajectories $y_{d 1}=\sin (t)$ and $y_{d 2}=\sin (t)$. Two square waves having an amplitude \pm 1 with a period of $2 \pi(s)$ are added to system states as external disturbances. Then, the system (92) becomes

$\ddot{y}=F(x)+G(x) \Phi(u)+d(t)$ 
Recall that the system nonlinearities $F(x)$ and $G(x)$ are assumed to be completely unknown except the symmetry property and the sign of the matrix $G(x)$ when designing the fuzzy adaptive controller.

The fuzzy systems $\theta_{1}^{T} \psi_{1}(x)$ and $\theta_{2}^{T} \psi_{2}(x)$ used to approximate the unknown nonlinearities are designed as (22) and have only the state vector $x=\left[q_{1}, \dot{q}_{1}, q_{2}, \dot{q}_{2}\right]^{T}$ as input. For each variable of the entries of the two fuzzy systems, as in [26], one defines three (one triangular and two trapezoidal) membership functions uniformly distributed on the interval $[-2,2]$. Thus, the number of the fuzzy rules is $3^{4}$ (because our fuzzy rule base is a completed base). The consequent parameters are estimated online via the adaptive laws (35), they are initialized as follows: $\theta_{1 i}(0)=\theta_{2 i}(0)=0.001$, for $i=1, \ldots, 81$.

The design parameters used in this simulation are specified as follows: $\gamma_{01}=\gamma_{02}=30, \quad \gamma_{11}=\gamma_{12}=700, \sigma_{01}=\sigma_{02}=0.001$, $\sigma_{11}=\sigma_{12}=0.001, \lambda_{1}=\lambda_{2}=2$ and $k_{11}=k_{12}=2$. The initial conditions are chosen as follows: $x(0)=\left[\begin{array}{llll}0.5 & 0 & 0.5 & 0\end{array}\right]^{T}$, and $k_{01}(0)=k_{02}(0)=0.0005$. It is worth noticing that the sign function has been replaced in the simulation by a smooth function $\tanh \left(k_{s i} S_{i}\right)$, with $k_{s i}=20$.

Figure 2 shows proposed fuzzy adaptive controller performs well in spite of the input nonlinearities, uncertainties and state disturbances. Indeed, the tracking errors for both links are bounded and converge towards small values as pointed out by Fig. 2a, b. Figure 2c, $d$ show that the control variables as well as the norms of the estimated parameters are bounded.

\subsubsection{Example 2 (Test of the second controller)}

In the following, one presents simulation results showing the performance of the second fuzzy adaptive control system involving an academic multivariable nonlinear system having a nonsymmetric and indefinite control-gain matrix. The dynamics of this system is given by:

$$
\left\{\begin{array}{l}
\dot{x}_{11}=x_{12}, \\
\dot{x}_{12}=x_{21}-0.3 \sin \left(x_{11} x_{12}\right)+x_{12}^{2}+\left(2+\cos \left(x_{11}\right)\right) \Phi_{1}\left(u_{1}\right)+\left(1+\left(\sin \left(x_{21}\right)\right)^{2}\right) \Phi_{2}\left(u_{2}\right), \\
\dot{x}_{21}=x_{22}, \\
\dot{x}_{22}=x_{22}^{3}+e^{x_{11}}+x_{12}^{2}-0.5 \Phi_{1}\left(u_{1}\right)-\left(1+\left(\sin \left(x_{21}\right)\right)^{2}\right) \Phi_{2}\left(u_{2}\right), \\
y_{1}=x_{11}, \quad y_{2}=x_{21} .
\end{array}\right.
$$

Let $y=\left[y_{1}, y_{2}\right]^{T}, \quad u=\left[u_{1}, u_{2}\right]^{T}, \Phi(u)=\left[\Phi_{1}\left(u_{1}\right), \Phi_{2}\left(u_{2}\right)\right]^{T}$, and $x=\left[x_{11}, x_{12}, x_{21}, x_{22}\right]^{T}$. Then, the system (94) can be given in the following form:

$\ddot{y}=F(x)+G(x) \Phi(u)$

where

$$
\begin{aligned}
& F(x)=\left(\begin{array}{cc}
x_{21}-0.3 \sin \left(x_{11} x_{12}\right)+x_{12}^{2} \\
x_{22}^{3}+e^{x_{11}}+x_{12}^{2}
\end{array}\right) \\
& G(x)=\left(\begin{array}{cc}
\left(2+\cos \left(x_{11}\right)\right) & 1+\left(\sin \left(x_{21}\right)\right)^{2} \\
-0.5 & -\left(1+\left(\sin \left(x_{21}\right)\right)\right)^{2}
\end{array}\right)
\end{aligned}
$$

The input nonlinearities $\Phi_{i}\left(u_{i}\right)$ for $i=1,2$ are described by:

$$
\Phi_{i}\left(u_{i}\right)=\left\{\begin{array}{lc}
\left(u_{i}-3\right)\left(1-0.3 \sin \left(u_{i}\right)\right), & u_{i}>3 \\
0, & -3 \leq u_{i} \leq 3 \\
\left(u_{i}+3\right)\left(0.8-0.3 \cos \left(u_{i}\right)\right), & u_{i}<-3
\end{array}\right.
$$

Recall that the matrix $G(x)$ is nonsymmetric and the system nonlinearities $(F(x)$ and $G(x))$ are assumed to be unknown except the signs of its leading principal minors when designing the fuzzy adaptive controller.

The control objective consists in allowing the system outputs $y_{1}$ and $y_{2}$ to track the sinusoidal desired trajectories $y_{d 1}=$ $\sin (t)$ and $y_{d 2}=\sin (t)$, respectively.

The fuzzy system $\theta_{1}^{T} \psi_{1}\left(\bar{z}_{1}\right)$ has the vector $\left[x^{T}, u_{2}\right]^{T}$ as input, while the fuzzy system $\theta_{2}^{T} \psi_{2}\left(\bar{z}_{2}\right)$ has the state vector $x$ as input. For each variable of the entries of these fuzzy systems, as in [26], one defines three (one triangular and two trapezoidal) membership functions uniformly distributed on the intervals [-2,2] for $x_{11}, x_{12}, x_{21}$, and $x_{22}$, and [-25,25] for $u_{2}$. Thus, the number of the fuzzy rules (for both fuzzy systems) is $3^{5}$ and $3^{4}$, respectively. Note that our fuzzy rule bases are completed bases. The consequent parameters are estimated online via the adaptive laws (64), they are initialized in a random way: $\theta_{1 i}(0)=0.001$, for $i=1, \ldots, 243$ and $\theta_{2 j}(0)=$ 0.002 , for $j=1, \ldots, 81$.

The design parameters used in this simulation are chosen as follows: $\gamma_{01}=\gamma_{02}=20, \quad \gamma_{11}=\gamma_{12}=1500, \sigma_{01}=\sigma_{02}=0.01$, $\sigma_{11}=0.001, \sigma_{12}=0.001, \lambda_{1}=\lambda_{2}=2$ and $k_{11}=k_{12}=0.5$. The initial conditions are selected as: $x(0)=\left[\begin{array}{llll}0.5 & 0 & 0.5 & 0\end{array}\right]^{T}$ and $k_{01}(0)=k_{02}(0)=0.001$,. The sign function has been replaced by an equivalent smooth function, i.e., $\tanh \left(k_{s i} \bar{S}_{i}\right)$, with $k_{s i}=20$. 
Fig. 2 Simulation results obtained by the controller 1 of the example 1. a Tracking errors of link 1: $e_{1}$ (dotted line) and $\dot{e}_{1}$ (solid line). b Tracking errors of link 2: $e_{2}$ (dotted line) and $\dot{e}_{2}$ (solid line). c Control input signals: $u_{1}$ (dotted line) and $u_{2}$ (solid line; d). Norm of fuzzy parameters: $\left\|\theta_{1}\right\|$ (dotted line), $\left\|\theta_{2}\right\|$ (solid line) a

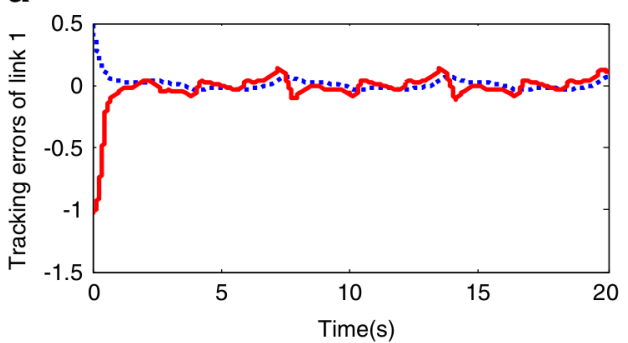

C

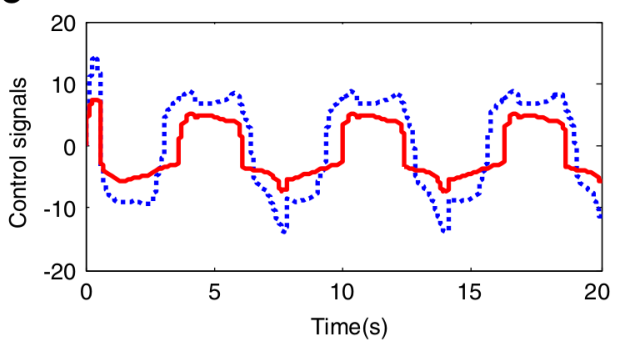

b

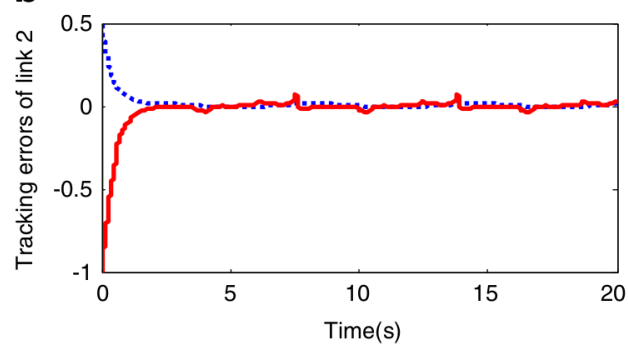

d

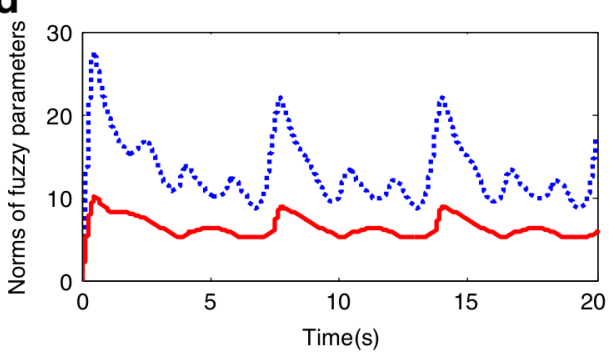

Figure 3 shows that the proposed fuzzy adaptive control system performs well from tracking point of view. In fact, Fig. 3a, b illustrate the boundedness and convergence of the tracking errors for both subsystems. The boundedness of the corresponding control signals as well as of the estimated fuzzy parameters is well illustrated in Fig. 3c, d, respectively. In spite of the presence of the unknown input nonlinearities (dead zone and sector nonlinearities) and the uncertainties, those simulation results show a good tracking performance.
5.2 Test of the fuzzy adaptive output feedback controller

\subsubsection{Example 1}

When the states $\left(x_{12}, x_{22}\right)$ of the system (92) are not measurable, a high-gain observer is designed as follows:

$$
\left\{\begin{array}{l}
\varepsilon \dot{\pi}_{11}=\pi_{12} \\
\varepsilon \dot{\pi}_{12}=-b_{11} \pi_{12}-\pi_{11}+y_{1} \\
\varepsilon \dot{\pi}_{21}=\pi_{22} \\
\varepsilon \dot{\pi}_{22}=-b_{21} \pi_{22}-\pi_{21}+y_{2}
\end{array}\right.
$$

Fig. 3 Simulation results obtained by the controller 2 of the example 2. a Tracking errors: $e_{1}$ (dotted line) and $\dot{e}_{1}$ (solid line). b Tracking errors: $e_{2}$ (dotted line) and $\dot{e}_{2}$ (solid line). c Control input signals: $u_{1}$ (dotted line) and $u_{2}$ (solid line; $\mathbf{d}$ ). Norm of fuzzy parameters: $\left\|\theta_{1}\right\|$ (dotted line), $\left\|\theta_{2}\right\|$ (solid line) a

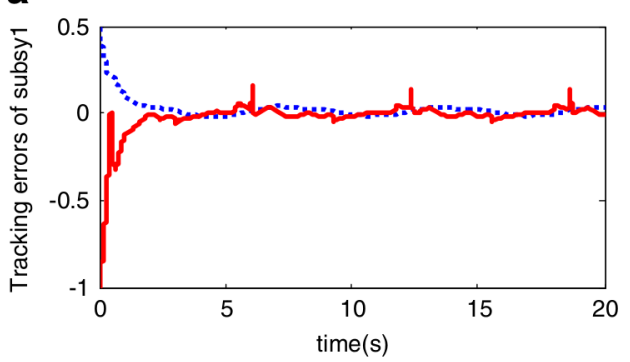

b

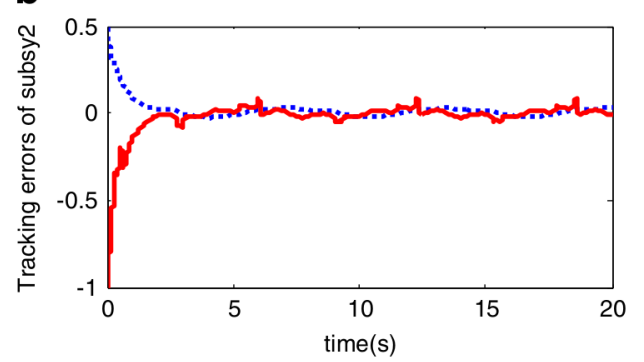

C

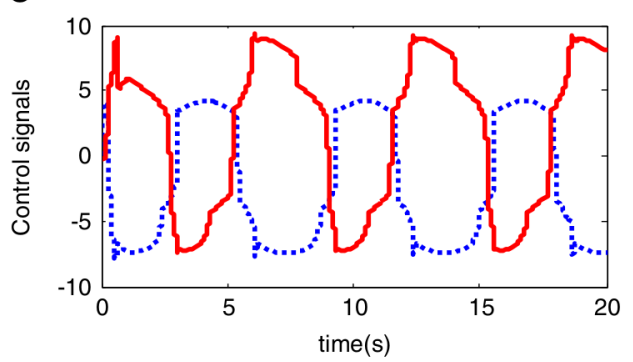

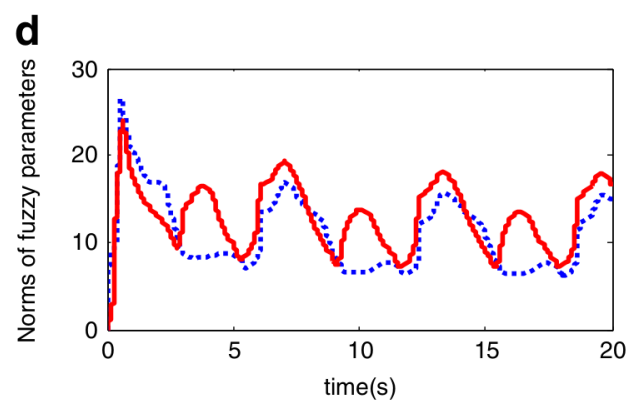


Fig. 4 Simulation results obtained by the controller 3 of the example 1 . a Tracking errors of link 1: $e_{1}$ (dotted line) and $\dot{e}_{1}$ (solid line). b Tracking errors of link 2: $e_{2}$ (dotted line) and $\dot{e}_{2}$ (solid line). c Control input signals: $u_{1}$ (dotted line) and $u_{2}$ (solid line; d). Observation errors: $\widetilde{e}_{12}=\widehat{x}_{12}-x_{12}$ (dotted line), $\widetilde{e}_{22}=\widehat{x}_{22}-x_{22}$ (solid line)
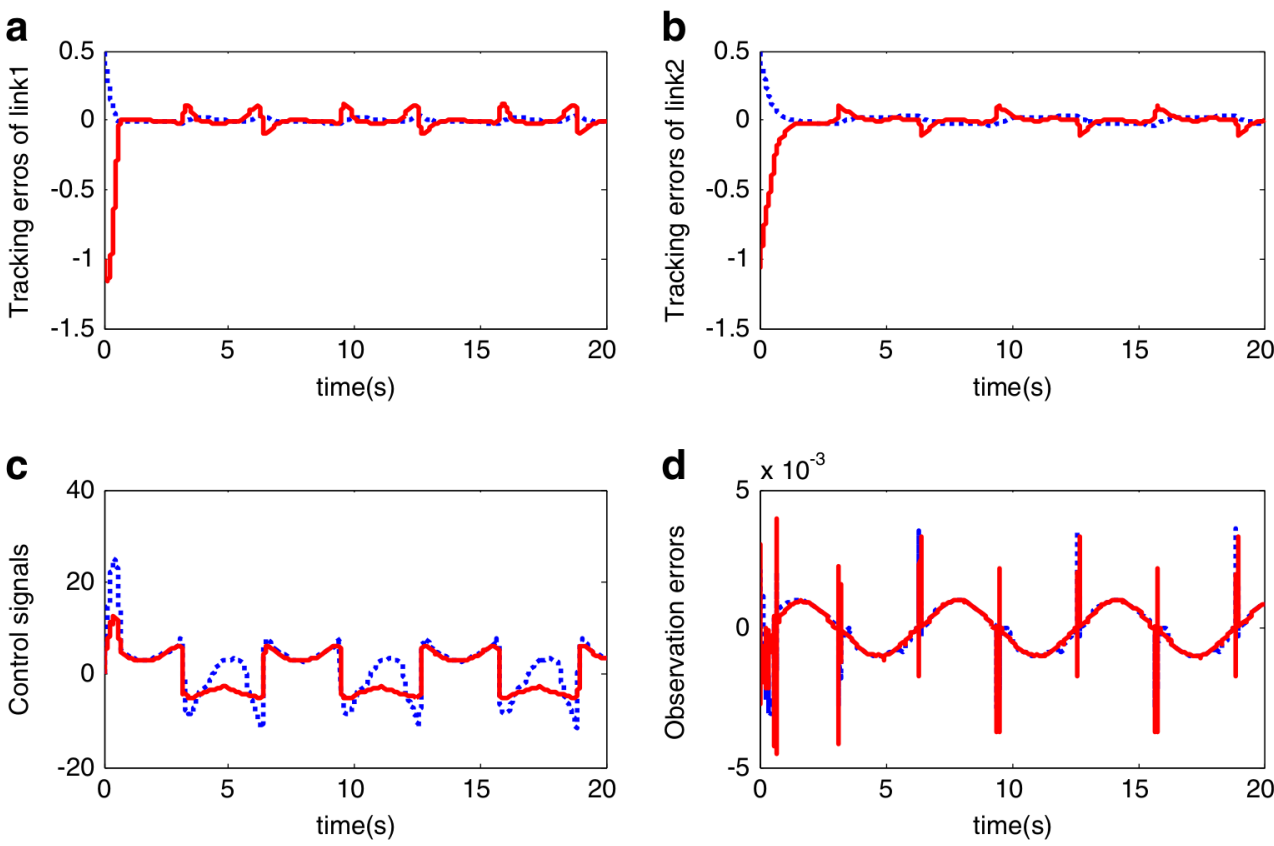

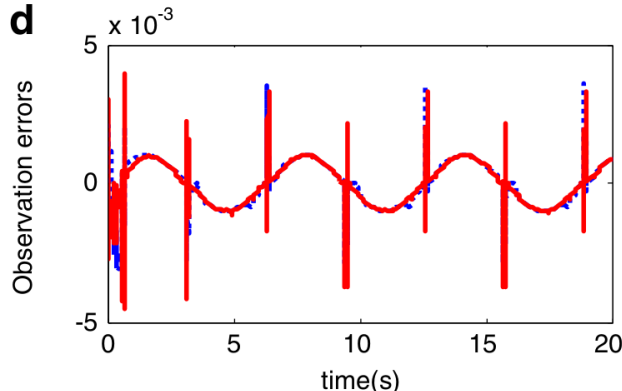

with $\varepsilon=0.01, b_{11}=b_{21}=1$, and the initial conditions $\pi(0)=[0,0,0,0]^{T}$. The respective estimates of the vectors $\bar{z}_{1}$ and $\bar{z}_{2}$ are:

$\widehat{\bar{z}}_{1}=\left[y_{1}, \frac{\pi_{12}}{\varepsilon}, \quad y_{2}, \frac{\pi_{22}}{\varepsilon}, u\right]^{T}$

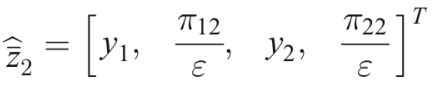

Figure 4 illustrates the simulation results of the fuzzy adaptive output feedback controller. Figure $4 a$, b show the boundedness and convergence of the tracking errors $\left(e_{1}, \dot{e}_{1}, e_{2}, \dot{e}_{2}\right)$ towards small values for both subsystems. Figure $4 \mathrm{c}$ indicates the boundedness of the applied control signals. Figure $4 \mathrm{~d}$ shows the observation errors $\left(\widetilde{e}_{12}=\widehat{x}_{12}-x_{12}\right.$, $\left.\widetilde{e}_{22}=\widehat{x}_{22}-x_{22}\right)$ are very small and bounded.

\subsubsection{Example 2}

When the states $\left(x_{12}, x_{22}\right)$ of the system (94) are not available for measurement, the high-gain observer (96) can be designed.
Fig. 5 Simulation results obtained by the controller 3 of the example 2 a Tracking errors: $e_{1}$ (dotted line) and $\dot{e}_{1}$ (solid line). b Tracking errors: $e_{2}$ (dotted line) and $\dot{e}_{2}$ (solid line). c Control input signals: $u_{1}$ (dotted line) and $u_{2}$ (solid line; d). Observation errors: $\widetilde{e}_{12}=\widehat{x}_{12}-x_{12}$ (dotted line), $\widetilde{e}_{22}=\widehat{x}_{22}-x_{22}$ (solid line)
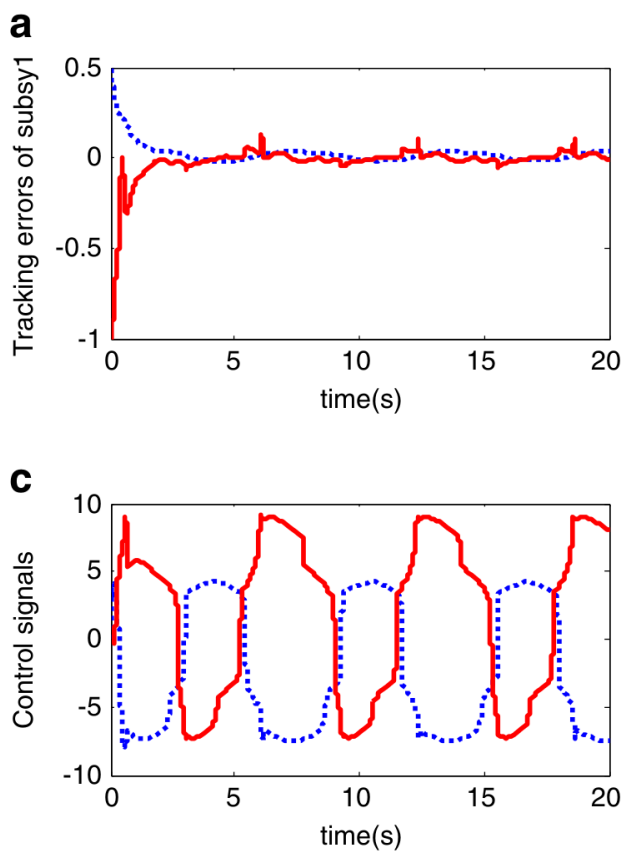

b
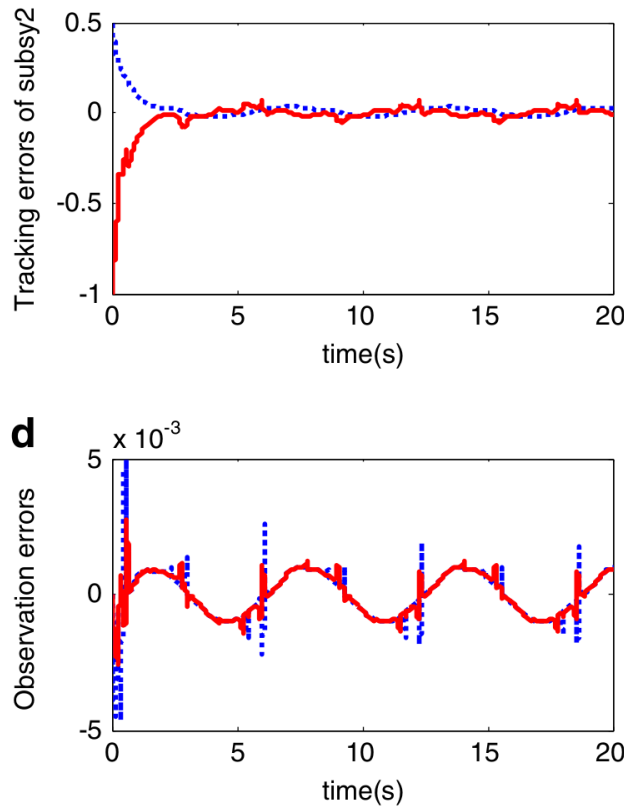
The parameters of this observer are selected as $\varepsilon=0.01$ and $b_{11}=b_{21}=1$, and the initial conditions as $\pi(0)=[0,0,0,0]^{T}$. The respective estimates of the vectors $\bar{z}_{1}$ and $\bar{z}_{2}$ can be determined as

$$
\begin{aligned}
& \widehat{\bar{z}}_{1}=\left[\begin{array}{llll}
y_{1}, & \frac{\pi_{12}}{\varepsilon}, & y_{2}, & \frac{\pi_{22}}{\varepsilon}, u
\end{array}\right]^{T} \\
& \widehat{\bar{z}}_{2}=\left[\begin{array}{llll}
y_{1}, & \frac{\pi_{12}}{\varepsilon}, & y_{2}, & \frac{\pi_{22}}{\varepsilon}
\end{array}\right]^{T}
\end{aligned}
$$

The simulation results of the fuzzy adaptive output feedback controller are given in Fig. 5. It can be seen in Fig. 5a, b, and $\mathrm{d}$ that tracking errors $\left(e_{1}, \dot{e}_{1}, e_{2}, \dot{e}_{2}\right)$ and observation errors $\left(\widetilde{e}_{12}=\widehat{x}_{12}-x_{12}, \widetilde{e}_{22}=\widehat{x}_{22}-x_{22}\right)$ are small. Figure $5 \mathrm{c}$ indicates the boundedness of applied control signals. The proposed output feedback controller achieves a satisfied tracking performance even with little knowledge of the system, in the presence of input nonlinearities and only the measurement of the output.

\section{Conclusion}

In this paper, three fuzzy adaptive controllers (the two first controllers are state feedback and but the last one is an output feedback) for a class of multivariable unknown nonlinear systems subject to actuator sector nonlinearities and dead zones have been proposed bearing in mind the usual stability and convergence requirements. The first one has been designed for systems having a symmetric and positive-definite control-gain matrix, while the second one has been particularly designed for systems with a nonsymmetric control gains matrix whose leading principal minors are non-zero. A suitable decomposition of the control-gain matrix has been fully exploited to carry out the second control design. The third one is an output feedback extension of the second controller. In the latter, a high-gain observer has been designed to estimate the missing states. Of fundamental interest, it has been proven that the proposed control systems are stable and their underlying tracking errors converge exponentially to an adjustable domain. Simulation results have been given to emphasize the effectiveness of the proposed controllers.

\section{References}

1. Wang LX (1994) Adaptive fuzzy systems and control: design and stability analysis. Prentice-Hall, Englewood Cliffs

2. Chang YC (2000) Robust tracking control for nonlinear MIMO systems via fuzzy approaches. Automatica 36:1535-1545

3. Chekireb H, Tadjine M, Bouchaffra D (2003) Direct adaptive fuzzy control of nonlinear system class with applications. Control Intell Syst 31(2):1-11
4. Essounbouli N, Hamzaoui A, Zaytoon J (2006) An improved robust adaptive fuzzy controller for MIMO systems. Control Intell Syst 34(1):12-21

5. Golea N, Golea A, Benmahammed K (2003) Stable indirect fuzzy adaptive control. Fuzzy Sets Syst 137:353-366

6. Labiod S, Boucherit MS, Guerra TM (2005) Adaptive fuzzy control of a class of MIMO nonlinear systems. Fuzzy Sets Syst 151:59-77

7. Li HX, Tong SC (2003) A hybrid adaptive fuzzy control for a class of nonlinear MIMO systems. IEEE Trans Fuzzy Syst 11(1):24-34

8. Ordonez R, Passino KM (1999) Stable multi-input multi-output adaptive fuzzy/neural control. IEEE Trans Fuzzy Syst 7(3):345-353

9. Tong SC, Tang J, Wang T (2000) Fuzzy adaptive control of multivariable nonlinear systems. Fuzzy Sets Syst 111(2):153-167

10. Tong SC, Li HX (2003) Fuzzy adaptive sliding model control for MIMO nonlinear systems. IEEE Trans Fuzzy Syst 11(3):354-360

11. Tong SC, Chen B, Wang Y (2005) Fuzzy adaptive output feedback control for MIMO nonlinear systems. Fuzzy Sets Syst 156(2):285299

12. Gutierrez HM, Ro PI (1998) Sliding-mode control of a nonlinearinput system: application to a magnetically levitated fast-tool servo. IEEE Trans Ind Electron 45:921-927

13. Hsu KC, Wang WY, Lin PZ (2004) Sliding mode control for uncertain nonlinear systems with multiple inputs containing sector nonlinearities and deadzones. IEEE Trans Syst Man Cybern Part-B 34(1): 374-380

14. Hsu KC (1999) Decentralized sliding mode controller for uncertain time-delayed systems with series nonlinearities. ASME J Dyn Syst Meas Control 121(4):708-713

15. Shyu KK, Liu WJ, Hsu KC (2003) Decentralized variable structure control design for uncertain large scale systems containing a deadzone. IEE Proc Control Theory Appl 150(5):467-475

16. Shyu KK, Liu WJ, Hsu KC (2005) Design of large-scale timedelayed systems with dead-zone input via variable structure control. Automatica 41:1239-1246

17. Niu Y, Ho DWC (2006) Design of sliding mode control for nonlinear stochastic systems subject to actuator nonlinearity. IEE Proc Control Theory Appl 153(6):737-744

18. Zhang TP, Ge SS (2007) Adaptive neural control of MIMO nonlinear state time-varying delay systems with unknown dead-zones and gain signs. Automatica 43(6):1021-1033

19. Zhang TP, Yi Y (2007) Adaptive fuzzy control for a class of MIMO nonlinear systems with unknown dead-zones. Acta Autom Sin 33(1): 96-99

20. Boulkroune A, M'Saad M, Tadjine M, Farza M (2008) Adaptive fuzzy control for MIMO nonlinear systems with unknown deadzone. Proc. 4th International IEEE Conference on Intelligent Systems, Varna, pp 450-455

21. Zhang M, Yu Z, Huan H, Zhou Y (2008) The sliding mode variable structure control based on composite reaching law of active magnetic bearing. ICIC Express Lett 2(1):59-63

22. Li C, Tong SC, Wang Y (2008) Fuzzy adaptive fault tolerant sliding mode control for SISO nonlinear systems. Int J Innov Comput Inf Control 4(12):3375-3384

23. Masuike H, Ikuta A (2009) Fuzzy adaptive filter for state estimation of sound environment system and its application to psychological evaluation. Int $\mathrm{J}$ Innov Comput Inf Control 5(12B): $4797-4808$

24. Zhang W, Wang T, Tong SC (2009) Delay-dependent stabilization conditions and control of T-S fuzzy systems with time-delay. ICIC Express Lett 3(4A):871-876

25. Boulkroune A, M'Saad M, Tadjine M, Farza M (2010) Fuzzy adaptive controller for MIMO nonlinear systems with known and unknown control direction. Fuzzy Sets Syst 161(3):797-820

26. Boulkroune A, Tadjine M, Msaad M, Farza M (2008) How to design a fuzzy adaptive control based on observers for uncertain affine nonlinear systems. Fuzzy Sets Syst 159:926-948 
27. Boulkroune A, Tadjine M, Msaad M, Farza M (2009) Adaptive fuzzy controller for non-affine systems with zero dynamics. Int J Syst Sci 40(4):367-382

28. Hua C, Guan X, Shi P (2006) Decentralized robust model reference adaptive control for interconnected time-delay systems. J Comput Appl Math 193:383-396

29. Zhang L, Shi P, Wang C, Gao H (2006) Robust H-infinity filtering for switched linear discrete-time systems with polytopic uncertainties. Int J Adapt Control Sig Process 20:291-304

30. Lin Z, Zhu Z, Xia Y, Wang S (2010) Adaptive control for missile systems with parameter uncertainty. ICIC Express Lett 4(5B):19371944

31. Qin P, Han X, Lin Y (2009) Convergence analysis about improved model-free adaptive controller based on multi-innovation theory. ICIC Express Lett 3(3A):301-306

32. Chen J, Behal A, Dawson DM (2006) Adaptive output feedback control for a class of MIMO nonlinear systems. Proc. of the American Control Conference, Minneapolis, pp 5300-5305

33. Costa RR, Hsu L, Imai AK, Kokotovic P (2003) Lyapunov-based adaptive control of MIMO systems. Automatica 39(7):1251-1257

34. Hsu L, Costa RR, Lizarralde F (2007) Lyapunov/passivity-based adaptive control of relative degree two MIMO systems with an application to visual servoing. IEEE Trans Autom Control 52(2): 364-371

35. Zhang XT, Dawson DM, De Queiroz MS, Xian B (2004) Adaptive control for a class of MIMO nonlinear systems with non-symmetric input matrix. Proc. of the IEEE International Conference on Control Applications, Taipei, pp 1324-1329
36. Strang G (1980) Linear algebra and its applications. Academic, New Work

37. Tong SC, Zhang W, Wang T (2010) Robust stabilization conditions and observer-based controllers for fuzzy systems with input delay. Int J Innov Comput Inf Control 6(12):5473-5484

38. Tong SC, Sheng N, Li Y (2011) Observer-based adaptive fuzzy backstepping control for strict-feedback nonlinear systems with unknown time delays. Int J of Inn Comput Inf Control 7(12):6949-6964

39. Ge SS, Zhang J (2004) Neural network control of nonaffine nonlinear system with zero dynamics by state and output feedback. IEEE Trans Neural Netw 14(4):900-918

40. Tee KP, Ge SS (2006) Control of fully actuated ocean surface vessels using a class of feedforward approximators. IEEE Trans Control Syst Technol 14(4):750-756

41. Behtash S (1990) Robust output tracking for nonlinear systems. Int J Control 51:1381-1405

42. Khalil HK (2001) Nonlinear systems, 3rd edn. Englewood Cliffs, Prentice-Hall

43. Shi P, Xia Y, Liu G, Rees D (2006) On designing of sliding mode control for stochastic jump systems. IEEE Trans Autom Control 51(1):97-103

44. Liu M, Shi P, Zhang L, Zhao X (2011) Fault tolerant control for nonlinear Markovian jump systems via proportional and derivative sliding mode observer. IEEE Trans Circ Syst I Reg Pap 58(11):27552764

45. Lin Z, Xia Y, Shi P, Wu H (2011) Robust sliding mode control for uncertain linear discrete systems independent of time-delay. Int J Innov Comput Inf Control 7(2):869-881 